\title{
Search for a heavy resonance decaying to a pair of vector bosons in the lepton plus merged jet final state at $\sqrt{s}=13 \mathrm{TeV}$
}

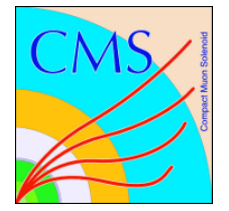

\section{The CMS collaboration}

\author{
E-mail: cms-publication-committee-chair@cern.ch
}

ABSTRACT: A search for a new heavy particle decaying to a pair of vector bosons (WW or WZ) is presented using data from the CMS detector corresponding to an integrated luminosity of $35.9 \mathrm{fb}^{-1}$ collected in proton-proton collisions at a centre-of-mass energy of $13 \mathrm{TeV}$ in 2016. One of the bosons is required to be a $\mathrm{W}$ boson decaying to $\mathrm{e} \nu$ or $\mu \nu$, while the other boson is required to be reconstructed as a single massive jet with substructure compatible with that of a highly-energetic quark pair from a $\mathrm{W}$ or $\mathrm{Z}$ boson decay. The search is performed in the resonance mass range between 1.0 and $4.4 \mathrm{TeV}$. The largest deviation from the background-only hypothesis is observed for a mass near $1.4 \mathrm{TeV}$ and corresponds to a local significance of 2.5 standard deviations. The result is interpreted as an upper bound on the resonance production cross section. Comparing the excluded cross section values and the expectations from theoretical calculations in the bulk graviton and heavy vector triplet models, spin- $2 \mathrm{WW}$ resonances with mass smaller than $1.07 \mathrm{TeV}$ and spin-1 WZ resonances lighter than $3.05 \mathrm{TeV}$, respectively, are excluded at $95 \%$ confidence level.

Keywords: Beyond Standard Model, Hadron-Hadron scattering (experiments)

ARXIV EPRINT: 1802.09407 


\section{Contents}

1 Introduction 1

2 The CMS detector 3

3 Benchmark signal models and simulated background samples 3

4 Event reconstruction and selection 4

$\begin{array}{llr}5 & \text { Signal extraction } & 7\end{array}$

$\begin{array}{llr}6 & \text { Systematic uncertainties } & 10\end{array}$

$\begin{array}{llr}7 & \text { Results } & 12\end{array}$

$\begin{array}{lll}8 & \text { Summary } & 14\end{array}$

$\begin{array}{ll}\text { The CMS collaboration } & 23\end{array}$

\section{Introduction}

The discovery of a Higgs boson at the CERN LHC [1-3] marked the success of fifty years of scientific investigation, during which the standard model (SM) [4-6] was first proposed and then consolidated with experimental evidence. However, an outstanding issue is the so-called hierarchy problem, i.e. the large difference between the energy scale at which electroweak symmetry breaks and the Planck scale at which gravity becomes important. Following a reasoning based on naturalness [7-9], which has been successful in guiding physics discoveries in the last century [10], physics effects beyond the standard model (BSM) are expected at the electroweak scale.

Different kinds of BSM mechanisms have been proposed to solve the hierarchy problem. Several of these models predict the existence of new heavy particles coupled to the vector bosons $\mathrm{V}=\mathrm{W}, \mathrm{Z}$. Examples include models based on extra spatial dimensions [11-14] or on a composite nature of the Higgs boson [15-18]. From previous searches at colliders [1933 and indirect bounds from precision measurements, the masses of these hypothetical particles (spin-2 bulk gravitons $\mathrm{G}_{\text {bulk }}$ and spin-1 $\mathrm{W}^{\prime}$ and $\mathrm{Z}^{\prime}$ bosons) are expected to be above $\approx 1 \mathrm{TeV}$ [34]. With such a large mass, the resonance decay would result in two bosons of high momentum, which would give rise to distinctive signatures in the LHC detectors. For instance, a high-momentum $\mathrm{W}$ boson decaying to leptons ( $\mathrm{W} \rightarrow \ell \nu$ with $\ell=\mathrm{e}, \mu$ ) would lead to the observation of a lepton aligned with the undetected neutrino. A vector boson decaying to a $\mathrm{q} \overline{\mathrm{q}}^{\left({ }^{\prime}\right)}$ pair would result in a single, massive jet, which could be identified using techniques that reveal the substructure of the jet. 


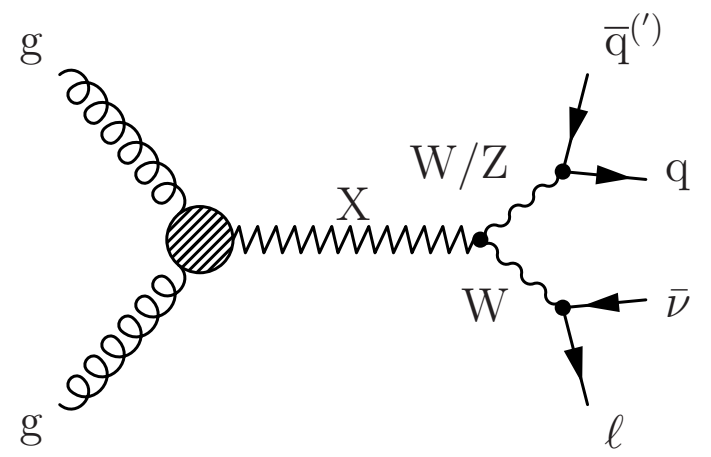

Figure 1. A Feynman diagram for the production of a generic resonance $\mathrm{X}$ decaying to the $\mathrm{WW} / \mathrm{WZ} \rightarrow \ell \nu \mathrm{q} \overline{\mathrm{q}}^{\left({ }^{\prime}\right)}$ final state.

In this paper, we describe a search for a heavy resonance decaying to a pair of vector bosons, one being a $\mathrm{W}$ boson decaying to an electron or muon and a neutrino, the other being a vector boson decaying to a $\mathrm{q} \overline{\mathrm{q}}^{\left({ }^{\prime}\right)}$ pair (see figure 1). The analysis is based on the proton-proton collision data set collected by the CMS experiment at the LHC in 2016, at a centre-of-mass energy of $13 \mathrm{TeV}$. The collected data correspond to an integrated luminosity of $35.9 \mathrm{fb}^{-1}$. Previous searches for these final states were performed by the ATLAS [30, 33, 35] and CMS [20, 25] Collaborations, resulting in stringent bounds on the masses of new resonances, e.g. $m_{\mathrm{W}^{\prime}}>2.99 \mathrm{TeV}$ for $\mathrm{W}^{\prime}$ particles in the heavy-vector triplet model B [35], but these searches have no sensitivity for bulk gravitons for the parameters used here (see section 3).

The dominant SM backgrounds to this search arise from lepton+jets events where the jets either originate from high-momentum vector boson decays to quark-antiquark pairs, $\mathrm{V} \rightarrow \mathrm{q} \overline{\mathrm{q}}^{\left({ }^{\prime}\right)}$, or are ordinary quark- or gluon-initiated jets. In this analysis, a new signal extraction method using a maximum likelihood fit is deployed, whereby the SM background contributions are estimated from data during the fit process. The fit is performed in the plane defined by the mass of the $\mathrm{V} \rightarrow \mathrm{q} \overline{\mathrm{q}}^{\left({ }^{\prime}\right)}$ boson candidate and the mass of the reconstructed diboson system. This two-dimensional (2D) approach further exploits the statistical power of the sideband and signal regions in a simultaneous fit, improving the discovery sensitivity across a large range of resonance masses. In addition, the new strategy increases the analysis flexibility, allowing a simultaneous search for WW and WZ resonances without having to focus on pre-defined jet mass search windows. The new method is checked against the previously employed background prediction method [20, 25], referred to as the $\alpha$ method.

This paper is organized as follows: the CMS detector is described in section 2. Section 3 introduces the BSM benchmark models utilized to interpret the result and the simulated samples used. Section 4 describes the event selection. Section 5 discusses the background model fit and its cross-check, based on the $\alpha$ method. The systematic uncertainties considered are given in section 6 . The interpretations of the analysis results in terms of benchmark BSM models are presented in section 7 . We conclude in section 8 . 


\section{The CMS detector}

The central feature of the CMS apparatus is a superconducting solenoid of $6 \mathrm{~m}$ internal diameter, providing a magnetic field of $3.8 \mathrm{~T}$. Within the solenoid volume are a silicon pixel and strip tracker, a lead tungstate crystal electromagnetic calorimeter (ECAL), and a brass and scintillator hadron calorimeter (HCAL), each composed of a barrel and two endcap sections. Forward calorimeters extend the pseudorapidity $(\eta)$ coverage provided by the barrel and endcap detectors. Muons are detected in gas-ionization chambers embedded in the steel flux-return yoke outside the solenoid. A more detailed description of the CMS detector, together with a definition of the coordinate system used and the relevant kinematic variables, can be found in ref. [36].

\section{Benchmark signal models and simulated background samples}

To interpret the results of this study, two BSM benchmark scenarios are considered: a spin2 bulk graviton model in which $\mathrm{G}_{\text {bulk }}$ decays to WW [13] and a heavy vector triplet (HVT) model with a charged spin-1 $\mathrm{W}^{\prime}$ decaying to WZ [34]. For the bulk graviton interpretation, the ratio of the unknown curvature scale of the extra dimension, $k$, and the reduced Planck mass, $\bar{M}_{\mathrm{Pl}} \equiv M_{\mathrm{Pl}} / \sqrt{8 \pi}$, is set to $\tilde{k} \equiv k / \bar{M}_{\mathrm{Pl}}=0.5$. This parameter choice ensures that the graviton natural width is negligible with respect to the experimental resolution (narrow-width approximation) [37]. The HVT model is a generic framework incorporating several models that predict additional gauge bosons, including composite Higgs models [18, 38-41], which are relevant to this analysis. The specific models are expressed in terms of a few parameters: the strength of the couplings to fermions, $c_{\mathrm{F}}$, the strength of the couplings to the Higgs boson and longitudinally polarized SM vector bosons, $c_{\mathrm{H}}$, and the interaction strength $g_{\mathrm{V}}$ of the new vector boson. For the analysis presented here, samples were simulated in HVT model B, corresponding to $g_{\mathrm{V}}=3, c_{\mathrm{H}}=-0.98$, and $c_{\mathrm{F}}=1.02$ [34]. For these parameters, the new resonances are narrow and have large branching fractions to boson pairs, while the fermionic couplings are suppressed. For each hypothesis, we consider resonance masses in the range 1.0-4.4 TeV. Simulated signal events are generated at leading order (LO) accuracy with MADGRAPH5_aMC@NLO v2.2.2 [42] with a relative resonance width of $0.1 \%$. The $\mathrm{LO}$ production cross section for $\mathrm{G}_{\mathrm{bulk}}$ resonances is rescaled by a mass-dependent $K$-factor, to match next-to-leading order (NLO) cross section values [37].

SM background samples are generated using Monte Carlo (MC) simulation. The $\mathrm{W}+$ jets process is simulated with MADGRAPH5_aMC@NLO at LO and normalized to the next-to-next-to-leading-order (NNLO) cross section, computed using FEWZ v3.1 [43]. The W boson transverse momentum $\left(p_{\mathrm{T}}\right)$ spectrum is corrected to account for NLO quantum chromodynamics (QCD) and electroweak contributions [44]. Top quark-antiquark (t $\overline{\mathrm{t}}$ ) events are generated with POWHEG v2 [45-50] and rescaled to the NNLO cross section value computed with Top ++ v2.0 [51]. Single top quark events are generated with both MADGRAPH5_aMC@NLO ( $s$-channel) and POWHEG (associated tW and $t$-channel production) at NLO, while diboson processes are generated at NLO with MADGRAPH5_aMC@NLO using the merging scheme in ref. [52] for WZ and ZZ, and with POWHEG for WW. The 
simulated single top quark and diboson background is normalized using inclusive cross sections calculated at NLO, or NNLO in QCD, where available, using MCFM v6.6 [53-55]. Parton showering and hadronization are implemented through PYTHIA v8.205 [56, 57] using the CUETP8M1 tune (CUETP8M2 for t' samples) [58, 59]. The NNPDF 3.0 [60] parton distribution functions (PDFs) are used for all simulated samples. All events are processed through a GEANT4-based [61] simulation of the CMS detector.

Simulated minimum bias interactions are added to the generated events to match the additional particle production observed in the large number of overlapping proton-proton interactions within the same or adjacent bunch crossings (pileup). The simulated events are weighted to reproduce the distribution of the number of pileup interactions observed in data, with an average of 21 reconstructed collisions per beam crossing. Furthermore, the simulated events are corrected for differences between data and simulation in the efficiencies of the triggers, lepton identification and isolation [62], and selection of jets originating from hadronization of $b$ quarks (b jets) [63].

\section{Event reconstruction and selection}

Event reconstruction is based on the particle-flow (PF) algorithm [64], which reconstructs and identifies each individual particle with an optimized combination of information from the various elements of the CMS detector. All events are required to have at least one primary vertex reconstructed within a $24 \mathrm{~cm}$ window along the beam axis, with a transverse distance from the nominal pp interaction region of less than $2 \mathrm{~cm}$ [65]. The reconstructed vertex with the largest value of summed physics-object $p_{\mathrm{T}}^{2}$ is taken to be the primary pp interaction vertex. The physics objects are the jets, clustered using the jet finding algorithm $[66,67]$ with the tracks assigned to the vertex as inputs, the charged leptons, and the associated missing transverse momentum, taken as the negative vector sum of the $p_{\mathrm{T}}$ of those jets and leptons.

The curvature of muon tracks is obtained by a global fit using measurements from the inner tracker and the muon detectors. The energy of electrons is determined from a combination of the electron momentum at the primary interaction vertex as determined by the tracker, the energy of the corresponding ECAL cluster, and the energy sum of all bremsstrahlung photons spatially compatible with originating from the electron track. The energy of charged hadrons is determined from a combination of their momentum measured in the tracker and the matching ECAL and HCAL energy deposits, corrected for zerosuppression effects and for the response function of the calorimeters to hadronic showers. The energy of neutral hadrons is obtained from the corresponding corrected ECAL and HCAL energy. The missing transverse momentum vector $\vec{p}_{\mathrm{T}}^{\text {miss }}$ is defined as the projection onto the plane perpendicular to the beam axis of the negative vector sum of the momenta of all reconstructed PF objects in the event, and its magnitude is denoted as $p_{\mathrm{T}}^{\text {miss }}$.

Events are selected by the trigger system [68] if a muon is present in the event with $p_{\mathrm{T}}>50 \mathrm{GeV}$ and $|\eta|<2.4$, or if an electron is identified within $|\eta|<2.5$ with thresholds of $p_{\mathrm{T}}>27,55$ and $105 \mathrm{GeV}$ for tight, loose, or no isolation criteria applied [69], respectively. In 
addition, events with $p_{\mathrm{T}}^{\text {miss }}>120 \mathrm{GeV}$ are included to further increase the trigger efficiency by exploiting the high $p_{\mathrm{T}}$ of the neutrino present in the leptonic $\mathrm{W}$ boson decay.

The offline muon and electron event selection requires $p_{\mathrm{T}}>55 \mathrm{GeV}$ with the same $\eta$-acceptance cuts as applied in the trigger. Requirements on lepton reconstruction quality and lepton identification are optimized to maintain a high reconstruction efficiency over the whole energy spectrum $[69,70]$. The muons are required to be isolated from other particles by requiring that the $p_{\mathrm{T}}$ sum of charged and pileup-corrected neutral particles in a cone of $\Delta R=\sqrt{(\Delta \eta)^{2}+(\Delta \phi)^{2}}=0.3$ (where the azimuthal angle $\phi$ is measured in radians) around the muon direction is less than $5 \%$ of the muon $p_{\mathrm{T}}$, to reject muons from heavy-flavour processes and decays in flight. For electrons, the selection cuts include requirements on the geometrical matching between ECAL depositions and the positions of reconstructed tracks, the ratio of the energies deposited in the HCAL and ECAL, the distribution of the ECAL depositions, and the number of reconstructed hits in the silicon tracker. Requirements on the impact parameters of electron and muon tracks with respect to the primary interaction vertex are applied to suppress the contributions from secondary decays and pileup interactions. Events with only one identified electron or muon are considered; those with additional muons (electrons) with $p_{\mathrm{T}}>20(35) \mathrm{GeV}$ are discarded.

Two kinds of jets are clustered: large-radius jets are formed by clustering the PF particles with the anti- $k_{\mathrm{T}}$ algorithm [66, 67] using a distance parameter $R=0.8$, while for standard jets $R=0.4$ is used. Large-radius jets with $p_{\mathrm{T}}>200 \mathrm{GeV}$ and standard jets with $p_{\mathrm{T}}>30 \mathrm{GeV}$ are considered. The jet momentum is determined as the vectorial sum of all particle momenta in the jet, and is found from simulation to be within 5 to $10 \%$ of the true momentum over the whole $p_{\mathrm{T}}$ spectrum and detector acceptance. Jet energy corrections are derived from simulation, and are confirmed with in situ measurements of the energy balance in dijet, multijet, $\gamma+$ jet, and leptonically decaying $Z+$ jets events [71]. To suppress jets originating from pileup interactions and to mitigate the impact of pileup on jet-related observables, we take advantage of the PUPPI algorithm [72], which uses local shape information to rescale the momentum of each particle according to its compatibility with the primary interaction vertex. Quality criteria are applied to the jets to remove spurious jet-like features originating from isolated noise [73]. In addition, global filters are applied to remove events with instrumental noise, which would result in artificially large values of $p_{\mathrm{T}}^{\text {miss }}[74]$. Both standard and large-radius jets are required to lie within the tracker acceptance of $|\eta|<2.5$ where the pileup jet identification and jet substructure algorithms have optimal performance. Since the signal is expected to be produced centrally, this angular requirement has no significant effect on the signal selection efficiency. Largeradius jets located within $\Delta R<1.0$ of a selected lepton are discarded, as well as standard jets located within $\Delta R<0.8$ of a large-radius jet or $\Delta R<0.4$ of a selected lepton.

Events of the muon (electron) channel are considered in the analysis if the $p_{\mathrm{T}}^{\text {miss }}$ in the event is greater than $40(80) \mathrm{GeV}$. The $\vec{p}_{\mathrm{T}}^{\text {miss }}$ is considered as an estimate of the $\vec{p}_{\mathrm{T}}$ of the neutrino coming from the $\mathrm{W}$ boson decay, and the longitudinal component $p_{z}$ of the neutrino momentum is estimated by imposing a $\mathrm{W}$ boson mass constraint to the lepton+neutrino system and solving the corresponding quadratic equation. The solution with smallest magnitude of the neutrino $p_{z}$ is considered. When no real solution is found, only 
the real part is considered. The leptonically decaying $\mathrm{W}$ boson candidate is then required to have $p_{\mathrm{T}}>200 \mathrm{GeV}$, and is combined with the most energetic large-radius jet in the event to form a WV resonance candidate.

In order to identify large-radius jets as Lorentz-boosted vector bosons, we define a $\mathrm{V}$ tagging algorithm, based on an estimate of the jet mass and the ratio of the $\mathrm{N}$ subjettiness [75] variables $\tau_{21}=\tau_{2} / \tau_{1}$. The jet mass is determined by applying a modified mass-drop algorithm [76, 77], known as the soft-drop algorithm [78], to large-radius jets, with parameters $\beta=0, z_{\text {cut }}=0.1$, and $R_{0}=0.8$. The $N$-subjettiness variables are computed fixing the values of the input parameters to $\beta=1.0$ and $R_{0}=0.8$. Jets coming from two-prong $\mathrm{W}$ or $\mathrm{Z}$ decays are characterized by lower values of $\tau_{21}$ than one-prong jets from SM backgrounds. Large-radius jets with soft-drop mass $m_{\text {jet }}$ between 30 and $210 \mathrm{GeV}$ and having $\tau_{21}<0.75$ are tagged as $\mathrm{V}$ jets. The use of a large window for $m_{\text {jet }}$ allows $\mathrm{W}, \mathrm{Z}$, and boosted top quark large-radius jet candidates to be selected, while retaining a sizeable low-mass sideband for background characterization.

Standard jets originating from $b$ quarks are identified by applying the combined secondary vertex algorithm (CSVv2) [63]. Events are rejected if a selected standard jet passes the medium working point of this algorithm. This working point has a probability for light-flavour jets (attributed to $\mathrm{u}, \mathrm{d}, \mathrm{s}$, or g partons) to be misidentified as b jets of about $1 \%$, and a $\mathrm{b}$ jet identification efficiency of about $70 \%$.

The lepton is required to be well separated from the $\mathrm{V}$-tagged jet, requiring an angular distance $\Delta R>\pi / 2$ between them. In addition, the difference in azimuthal angle between the $\mathrm{V}$-tagged jet and both the $\vec{p}_{\mathrm{T}}^{\text {miss }}$ and the $\mathrm{W} \rightarrow \ell \nu$ boson candidate directions is required to be $\Delta \phi>2$. The diboson mass $m_{\mathrm{WV}}$ is computed from the sum of the four-momenta of the $\mathrm{W} \rightarrow \ell \nu$ boson candidate and the V-tagged jet. Events with $m_{\mathrm{WV}}>800 \mathrm{GeV}$, for which a monotonically decreasing $m_{\mathrm{WV}}$ spectrum is guaranteed, are considered in the analysis. The overall selection efficiency times acceptance ranges from 47 to $57 \%$ for the $\mathrm{G}_{\text {bulk }}$ signal, and from 45 to $60 \%$ for the $\mathrm{W}^{\prime}$ signal, increasing with resonance mass.

The selected sample is separated into four mutually exclusive categories. First, the sample is split by lepton flavour, distinguishing muon from electron events. This facilitates accounting for the differences introduced by the different lepton reconstruction and selection. Subsequently, events are classified as high-purity (HP) or low-purity (LP), by requiring the $\mathrm{V}$-tagged jet to have $\tau_{21} \leq 0.55$ or $0.55<\tau_{21} \leq 0.75$, respectively. The definition of the HP and LP event categories was optimized by maximizing the expected significance for a bulk graviton signal over the full mass range using simulated events with $65<m_{\text {jet }}<105 \mathrm{GeV}$.

A control sample of $t \bar{t}$ events with similar kinematic distributions to the events in the signal region is selected by inverting the $\mathrm{b}$ jet veto. This sample is used to quantify the agreement between data and simulation in describing the $m_{\text {jet }}$ and $\tau_{21}$ variables. Figure 2 shows the distributions of these variables in the b-enriched sample, as an example in the electron channel. The observed disagreement between data and simulation is reasonable, since both variables are sensitive to hadronization, which is difficult to model. After applying the jet mass window and $\tau_{21}$ selection cuts, this sample is used to derive scale factors for the efficiency of the $\tau_{21}$ selection, and the resolution and scale of the jet mass peak. 

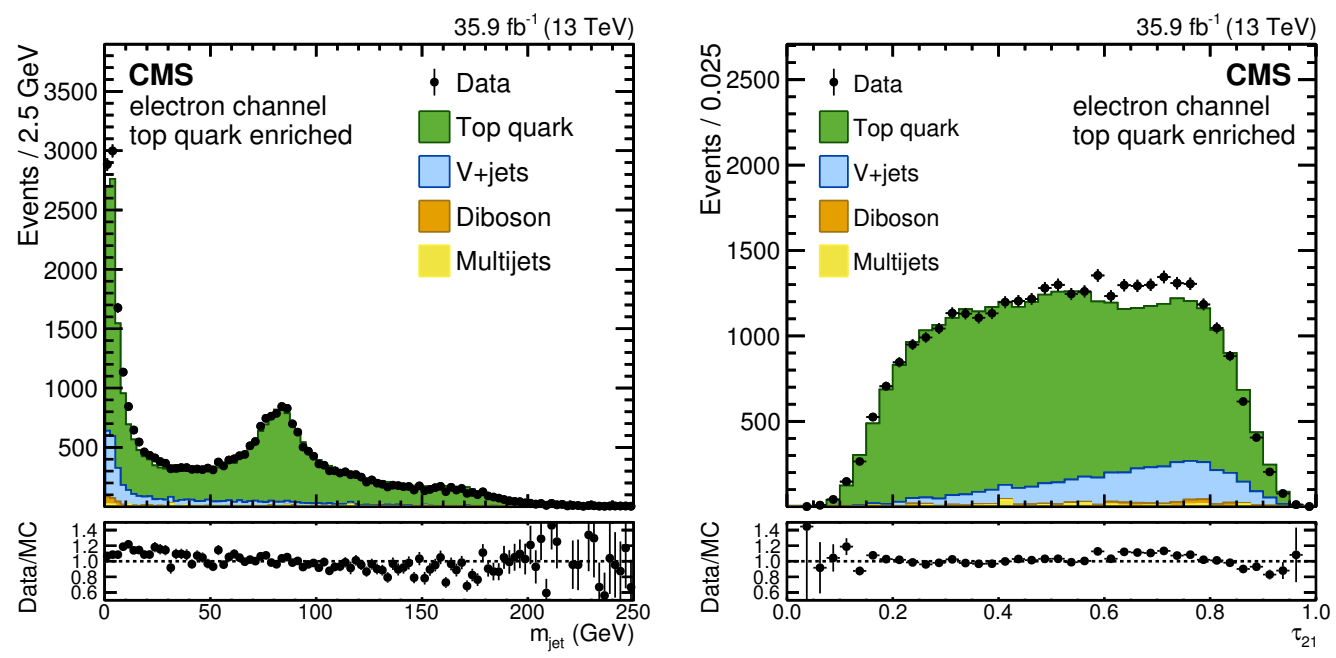

Figure 2. Jet soft-drop mass (left) and $N$-subjettiness ratio $\tau_{21}$ (right) for data and simulated events in the top quark enriched region in the electron channel. The contribution labelled as "Top

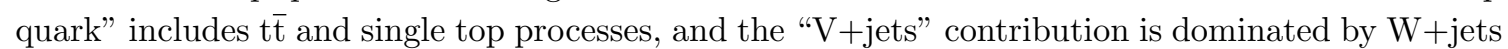
events with a small contribution from $\mathrm{Z}+$ jets events. The vertical bars correspond to the statistical uncertainties of the data.

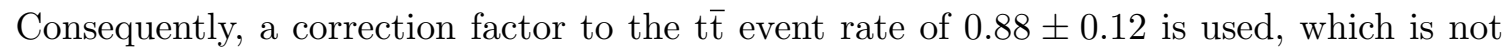
applied in figure 2 .

\section{Signal extraction}

For this analysis, a novel signal extraction method based on a 2D maximum likelihood fit is introduced. As a cross-check, the prediction obtained with the $\alpha$ method, used in previous versions of this analysis [20, 25], is also presented.

The signal and background yields are determined through a maximum likelihood fit, performed in the portion of the $\left(m_{\mathrm{WV}}, m_{\text {jet }}\right)$ plane defined by the event selection described in section 4 . The fit is performed using $2 \mathrm{D}$ templates for signal and background processes, starting from simulation and introducing shape uncertainties that model the difference between data and simulation in the full search range.

The probability density function (pdf) of $X \rightarrow \mathrm{WV}$ events in the $\left(m_{\mathrm{WV}}, m_{\text {jet }}\right)$ plane is modelled as:

$$
P_{\mathrm{sig}}\left(m_{\mathrm{WV}}, m_{\mathrm{jet}} \mid m_{\mathrm{X}}\right)=P_{\mathrm{WV}}\left(m_{\mathrm{WV}} \mid m_{\mathrm{X}}, \theta_{1}\right) P_{j}\left(m_{\mathrm{jet}} \mid m_{\mathrm{X}}, \theta_{2}\right)
$$

The pdfs $P_{\mathrm{WV}}$ and $P_{j}$ are represented by double Crystal Ball $[79,80]$ functions, and an additional exponential function is used in the jet mass dimension in LP events to model the tails of the soft-drop jet mass distribution. The parameters of the functions are described by uncorrelated polynomial interpolations, obtained by fitting the simulated signal sample distributions with the pdfs for different values of the resonance mass $m_{\mathrm{X}}$. The experimental resolution for $m_{\text {jet }}$ is around $10 \%$, and for $m_{\mathrm{WV}}$ it ranges from $6 \%$ at $1 \mathrm{TeV}$ to $4 \%$ at $4 \mathrm{TeV}$. 
For the signal, the dependence of the shape parameters on the resonance mass is found to depend on the nature of the $\mathrm{V}$ jet (e.g. $\mathrm{W}$ or $\mathrm{Z}$ ) and the lepton flavour. The signal yields in the different signal categories are expressed as a function of the integrated luminosity of the sample and the product of the signal acceptance and efficiency, treated as nuisance parameters, so that the resonance production cross section is determined in a combined fit to data in the four categories.

Two classes of background events are considered:

i. A W+jets background, consisting of a lepton and at least one jet arising from a quark or gluon mistagged as a $\mathrm{V}$ jet. In addition to $\mathrm{W} \rightarrow \ell \nu+$ jets, this background also includes $t \bar{t}$ production where the leptonically decaying $\mathrm{W}$ boson was reconstructed, but the merged jet corresponds to a random combination of jets in the event and not to a $\mathrm{W}$ boson or a top quark decay.

ii. A W+V/t background, peaking in $m_{\text {jet }}$ while smoothly falling in $m_{\mathrm{WV}}$. This background is dominated by t $\overline{\mathrm{t}}$ production while sub-dominant contributions include SM diboson and single top production.

Each background is modelled by a separate shape pdf based on its properties.

The $\mathrm{W}+$ jets background shape is described as a conditional probability of $m_{\mathrm{WV}}$ as a function of $m_{\text {jet }}$ :

$$
P_{\mathrm{W}+\text { jets }}\left(m_{\mathrm{WV}}, m_{\mathrm{jet}}\right)=P_{\mathrm{WV}}\left(m_{\mathrm{WV}} \mid m_{\text {jet }}, \theta_{1}\right) P_{j}\left(m_{\mathrm{jet}} \mid \theta_{2}\right) .
$$

The conditional probability is essential to take into account the large correlations between $m_{\text {jet }}$ and $m_{\mathrm{WV}}$. Those correlations arise from the strong dependence of the jet mass on the jet $p_{\mathrm{T}}$ during the hadronization process. The $2 \mathrm{D}$ conditional templates, $P_{\mathrm{WV}}$, are constructed from simulated events, starting before the detector simulation stage. For each event in the background samples, jets are clustered from stable particles using the same substructure algorithms as during event reconstruction. Consequently, a scale and resolution model is derived for both $m_{\text {jet }}$ and $m_{\mathrm{WV}}$ as a function of generated jet $p_{\mathrm{T}}$ by comparing the reconstructed and generated variables. Smooth templates are then populated as sums of 2D Gaussian distributions, where the mean values of the Gaussians correspond to the true value of $m_{\text {jet }}$ and $m_{\mathrm{WV}}$, shifted by the derived scale model, and the $2 \mathrm{D}$ covariance matrix is given by the resolution model. This technique is similar to the kernel-estimation procedure given in ref. [81] but uses the simulation and the exact resolution model instead of starting from reconstructed events. The final step is to smooth the tails for high values of $m_{\mathrm{WV}}$ ensuring there are no empty bins in the templates. The smoothing is performed by fitting events in each $m_{\text {jet }}$ bin with $m_{\mathrm{WV}}>2.0 \mathrm{TeV}$ using an exponential function and then using the function values to populate the tails for $m_{\mathrm{WV}}>2.5 \mathrm{TeV}$. The $P_{j}$ shapes are one-dimensional (1D) histograms derived directly from reconstructed simulated events, in contrast to the $P_{\mathrm{WV}}$ shapes discussed above.

For both the $P_{j}$ and $P_{\mathrm{WV}}$ components, nuisance parameters are introduced to account for differences between data and simulation. The most important difference is attributed to the different $p_{\mathrm{T}}$ spectrum of the jets in the simulation. The template construction is 
repeated by adding event weights corresponding to a harder (softer) spectrum, and the pdf is interpolated between these alternative templates. An additional uncertainty lies in the choice of the scale/resolution model, which is estimated by varying the scale as functions of $m_{\text {jet }}$ and $m_{\mathrm{WV}}$. The derived shapes are found to be in agreement with the simulated events, validating the template construction procedure. This procedure implicitly assumes

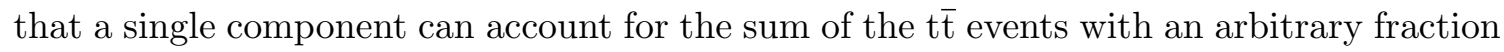
of reconstructed $\mathrm{W}+\mathrm{V}$ jet and $\mathrm{W}+$ jets contributions. A variation of the relative fractions is found to translate into a change in the average $p_{\mathrm{T}}$ spectrum, which is taken into account as a systematic uncertainty.

The $\mathrm{W}+\mathrm{V} / \mathrm{t}$ background is modelled as:

$$
P_{\mathrm{W}+\mathrm{V} / \mathrm{t}}\left(m_{\mathrm{WV}}, m_{\mathrm{jet}}\right)=P_{\mathrm{WV}}\left(m_{\mathrm{WV}} \mid \theta_{1}\right) P_{j}\left(m_{\mathrm{jet}} \mid m_{\mathrm{WV}}, \theta_{2}\right) .
$$

In this case, $P_{\mathrm{WV}}$ is a $1 \mathrm{D}$ template constructed in the same way as for the $\mathrm{W}+$ jets background, and the smoothing of its tail with an exponential function is performed for $m_{\mathrm{WV}}>1.2 \mathrm{TeV} . P_{j}$ is described by two peaks: a peak around the $\mathrm{W}$ boson mass dominated by top quark events where only the $\mathrm{W} \rightarrow \mathrm{q} \overline{\mathrm{q}}^{\prime}$ was reconstructed inside the large-radius jet, and a peak around the top quark mass where the $\mathrm{W}$ boson and the $\mathrm{b}$ quark decays are merged. These peaks are modelled by two double Crystal Ball plus one exponential function, whose parameters are described by uncorrelated polynomial functions of $m_{\mathrm{Wv}}$. The presence of both jet peaks allows additional scrutiny, since the relative fraction of the two peaks as a function of the resonance mass provides a robust validation of the top quark $p_{\mathrm{T}}$ spectrum convolved with effects from jet grooming. Different shapes are used in the individual event categories to account for differences in the event kinematic distributions. The $m_{\text {jet }}$ distribution for the $\mathrm{W}+\mathrm{V} / \mathrm{t}$ background in the region of the $\mathrm{W}$ boson peak is found to differ from the corresponding signal distribution. Indeed, this background component mainly consists of high-momentum t $\bar{t}$ events, in which a part of the $b$ jet from the $t \rightarrow \mathrm{Wb}$ decay overlaps with the $\mathrm{V}$ jet from the $\mathrm{W} \rightarrow \mathrm{q} \overline{\mathrm{q}}^{\prime}$ decay. This special kinematic configuration induces a modification in the $m_{\text {jet }}$ shape, which is taken into account using different functions to describe the $m_{\text {jet }}$ distributions of the signal and the $\mathrm{W}+\mathrm{V} / \mathrm{t}$ background.

The background estimation method employed in previous versions of this analysis is also applied, to cross-check the novel fit method. A full description of this $\alpha$ method, used to estimate the $\mathrm{W}+$ jets background from data, is presented in refs. [20, 25]. An unbinned fit to the $m_{\mathrm{WV}}$ distribution in data is performed, for events with $40 \leq m_{\text {jet }}<65 \mathrm{GeV}$ or $135 \leq m_{\text {jet }}<150 \mathrm{GeV}$. In this fit, the other background processes ( $\mathrm{t} \overline{\mathrm{t}}$, single top quark, etc.), which are modelled using functional shapes, are fixed to the prediction from simulated samples. Using a transfer factor $\alpha\left(m_{\mathrm{WV}}\right)$, estimated from a ratio derived from simulated samples, the result of the $m_{\text {jet }}$ sideband fit is extrapolated to the $\mathrm{W}$ and $\mathrm{Z}$ signal regions, defined by requiring $65 \leq m_{\text {jet }}<85 \mathrm{GeV}$ and $85 \leq m_{\text {jet }}<105 \mathrm{GeV}$, respectively. The separate $\mathrm{W}$ and $\mathrm{Z}$ boson mass windows double the number of signal categories to eight for the $\alpha$ method. The 2D fit approach provides an improvement to the expected sensitivity of the search by $20 \%$ compared to the $\alpha$ method, relying exclusively on data to predict the shape and the normalization of the backgrounds. 


\section{Systematic uncertainties}

Several systematic uncertainties affect the overall normalization and shape of the signal and backgrounds. Each effect is modelled by a nuisance parameter, which is profiled in the likelihood minimization. When specified, the uncertainty size represents the width of the function used to constrain the nuisance parameter (a log-normal function for systematic uncertainties related to normalization, and Gaussian functions for shape uncertainties).

The signal shape for the $2 \mathrm{D}$ fit in the $\left(m_{\text {jet }}, m_{\mathrm{WV}}\right)$ plane is affected by several systematic uncertainties: the jet energy scale ( $2 \%$ in the $m_{\mathrm{WV}}$ peak position) and resolution ( $5 \%$ in the $m_{\mathrm{WV}}$ peak width), $p_{\mathrm{T}}^{\text {miss }}$ energy scale and resolution $\left(2 \%\right.$ in both the $m_{\mathrm{WV}}$ peak position and width). Additional nuisance parameters are introduced to allow for variations of the soft-drop jet mass peak due to the effects of grooming on the scale and the resolution: a jet mass scale uncertainty of $1 \%$ and a resolution uncertainty of $2 \%$ are applied. Both values were estimated by fitting the $\mathrm{W}$ peak from $t \bar{t}$ events in an orthogonal control region defined by requiring the presence of a b-tagged jet. The jet mass scale and resolution are correlated across all resonant components including the signal and the $\mathrm{W}+\mathrm{V} / \mathrm{t}$ background.

The signal modelling for both methods is furthermore subject to uncertainties in the lepton modelling, as the uncertainty in the lepton energy scale is correlated with the obtained signal efficiency. Changes in lepton energy are propagated to the reconstructed $p_{T}^{\text {miss }}$, and through the entire analysis. The relative change in the number of selected signal events is taken as a systematic uncertainty in the signal normalization. These uncertainties are smaller than $1 \%$ for both lepton flavours, and are uncorrelated for different lepton flavours. In addition, the induced change in the peak position and width are added as systematic uncertainties in the distribution of the signal with an effect below 1\%. The systematic uncertainties in the lepton trigger, identification, and isolation efficiencies are obtained using a "tag-and-probe" method in $\mathrm{Z} \rightarrow \mathrm{ee} / \mu \mu$ events [62]. An uncertainty of $1-3 \%$ is assigned to the trigger efficiency for both lepton flavours, depending on the lepton $p_{\mathrm{T}}$ and $\eta$. For identification and isolation efficiencies, the systematic uncertainty is estimated to be $1-2 \%$ for muons and $3 \%$ for electrons.

A $2.5 \%$ uncertainty in the integrated luminosity [82] applies to the normalization of signal events. Uncertainties in the signal yield due to the choice of PDFs and factorization and renormalization scales are also taken into account by quantifying the change in acceptance. The scale uncertainties are evaluated following the proposal in refs. [83, 84]. The PDF uncertainties are evaluated using the NNPDF 3.0 [60] PDF set. The resulting uncertainties in acceptance are found to be negligible for the scale variation and range from 0.1 to $2 \%$ for the PDF evaluation. The signal cross section uncertainty arising from the uncertainty in PDFs and scales is not taken into account in the statistical analysis, but instead considered as an uncertainty in the theoretical cross section. These cross section uncertainties vary from 4 to $77 \%$ and from 2 to $23 \%$, respectively, depending on the resonance mass, particle type, and its production mechanism.

The background normalization and shape are estimated in the fit process. Therefore, large a priori uncertainties are assigned to the corresponding nuisance parameters, which are then constrained by the data. For the $\mathrm{W}+$ jets background, a normalization uncertainty 
of $50 \%$ is assumed, even though the observed difference between data and simulation when normalizing to integrated luminosity is significantly smaller. This large a priori uncertainty has no impact on the sensitivity; it provides a loose initial constraint for the fit, which precisely derives the normalization. For the $\mathrm{W}+\mathrm{V} / \mathrm{t}$ background, which is dominated by t $\overline{\mathrm{t}}$ production, a normalization uncertainty of $20 \%$ is assigned. Data and simulation in the top quark enriched region defined by inverting the $b$ jet veto agree to better than $10 \%$. The background normalization uncertainties are not correlated across the different categories.

Further systematic uncertainties lie in the $\mathrm{W}+$ jets and $\mathrm{W}+\mathrm{V} / \mathrm{t} m_{\mathrm{WV}}$ background shapes derived by the template-building method described in section 5 . These uncertainties are encoded in alternative shape functions, derived by repeating the template construction for different assumptions on the jet $p_{\mathrm{T}}$ spectrum as well as on the jet and resonance mass scale and resolution. One nuisance parameter is used to account for potential shifts of the $m_{\mathrm{WV}}$ spectrum due to uncertainties in the jet $p_{\mathrm{T}}$ spectrum: each bin along the $m_{\mathrm{WV}}$ direction is shifted by $\pm 0.1 m_{\mathrm{WV}} / \mathrm{TeV}$ to create mirrored templates in both directions. Similarly, another parameter is motivated by the measured dependence of the $m_{\mathrm{WV}}$ scale as a function of jet mass, resulting in a bin-by-bin shift of $\pm 400 \mathrm{GeV} / m_{\mathrm{WV}}$. The softdrop jet mass shape can be affected by additional physics effects specific to hadronization and jet substructure: two additional nuisance parameters are therefore used, one changing the shape by $\pm 0.002 m_{\text {jet }} / \mathrm{GeV}$ and another one by $\pm 15 \mathrm{GeV} / m_{\text {jet }}$. The values of these coefficients are chosen such that the resulting alternative shapes cover any differences between data and simulation observed in control regions. Several tests were performed with these variations and it was found that adding further parameters does not introduce any significant bias in the signal yields.

The $\mathrm{W}+\mathrm{V} / \mathrm{t}$ soft-drop jet mass shape is varied by scale and resolution uncertainties as is done for the signal. Additionally, a variation of the relevant fraction of the resonant $\mathrm{W}$ boson and top quark mass peaks is taken into account. By fitting the simulated distributions, we observed that the fraction of the two peaks can be modelled as:

$$
f=a+b / m_{\mathrm{WV}}^{2} .
$$

Two nuisance parameters are introduced to model the change of the top quark $p_{\mathrm{T}}$ spectrum, allowing a variation of $a$ by 0.2 and of $b$ by $25000 \mathrm{GeV}^{2}$. With these two parameters, the $p_{\mathrm{T}}$ spectrum of the top quark can be varied by about $30 \%$, constraining the $\mathrm{W}$ boson and top quark jet mass peaks.

The event categorization based on jet substructure introduces two additional sources of systematic uncertainties, namely the efficiency associated with the $\tau_{21}$ requirement (HP: 14\%, LP: $33 \%$ ) and the dependence of this efficiency on the jet $p_{\mathrm{T}}(4-13 \%)$. Both effects introduce a migration of events between the LP and HP categories. In total, 55 independent nuisance parameters are considered in the $2 \mathrm{D}$ fit, 14 of which affect the normalization of the signal and backgrounds, while 41 affect their shapes.

When applying the $\alpha$ method, all the normalization uncertainties listed above are taken into account. The uncertainty in the $m_{\mathrm{WV}}$ distribution is dominated by the statistical uncertainties in the simultaneous fits to the data of the sideband region, and the simulation in sideband and signal regions. An effect of almost equal magnitude arises from 
the uncertainties in the modelling of the transfer function $\alpha\left(m_{\mathrm{WV}}\right)$ between the sideband and the signal region. The uncertainty in the normalization of the background has three sources: the $\mathrm{W}+$ jets component, dominated by the statistical uncertainty of the events in the $m_{\text {jet }}$ sideband, varying from 2 to $6 \%$; the t $\bar{t} /$ single top quark component, dominated by the scale factor obtained from the top quark enriched control region, amounting to about $1-3 \%$, and by the $\mathrm{b}$ tagging scale factor, contributing $2-3 \%$; and the diboson component, dominated by the $\mathrm{V}$ tagging uncertainty, which varies in the range of $15-35 \%$.

\section{Results}

For both signal extraction techniques, the fit is performed simultaneously for the purity and lepton flavour categories. The result of the two-dimensional fit is presented in figures 3 and 4 , where projections in $m_{\text {jet }}$ and $m_{\mathrm{WV}}$ are shown for the HP and LP categories, respectively. The inclusive jet mass distributions demonstrate excellent modelling of both the resonant peaks and the continuum for all categories. In the LP category, the resonant background is dominated by the merged top quark contribution. These events show mostly a three-prong structure where both the quarks from the $\mathrm{W}$ boson and the $\mathrm{b}$ quark are resolved inside the large-radius jet, which makes it fail the $\tau_{21} \mathrm{HP}$ requirement.

Good modelling of the data is also observed in the resonance mass projections, where the $m_{\mathrm{WV}}$ distribution is plotted for events in the full jet mass range. The best fit values of the nuisance parameters are found within the range initially associated with each uncertainty. The quality of the fit is also quantified by forming a goodness-of-fit estimator using the saturated model [85]. The observed value of the estimator is compared with toy experiments and falls within the $68 \%$ interval containing the most probable results.

The search for new WW and WZ resonances is performed in the range of the resonance mass hypothesis between 1.0 and $4.4 \mathrm{TeV}$, which ensures that a hypothetical signal bump is contained within the $m_{\mathrm{WV}}$ search region ranging from 0.8 to $5.0 \mathrm{TeV}$. The data were also analyzed with the $\alpha$ method using separate $\mathrm{W}$ and $\mathrm{Z}$ boson mass windows. The results are found to be consistent between the two methods within their respective uncertainties, taking into account correlations.

Figure 5 shows a summary of the contributions of all categories to the signal extraction. Each event is weighted by the fraction of the number of expected signal events (S) over the sum of the expected signal and background events $(\mathrm{S}+\mathrm{B})$ in each category and in each soft-drop jet mass bin. The signal is hereby normalized to the production cross section of a graviton or $\mathrm{W}^{\prime}$ of mass $2 \mathrm{TeV}$ as predicted by the bulk graviton and HVT models, respectively, with parameters as defined in section 3 .

The largest deviation from the background hypothesis is observed for a mass hypothesis around $1.4 \mathrm{TeV}$ with a local significance of $2.4(2.5)$ standard deviations for the WW (WZ) signal, while the global significance in the search range is found to be 1.2 (1.4) standard deviations.

Several additional checks were pursued to validate the new background estimation technique. The range of the fit was reduced, starting at 1.0 instead of $0.8 \mathrm{TeV}$. The lower $m_{\mathrm{WV}}$ region is very important to constrain the top quark background around the $\mathrm{W}$ jet 

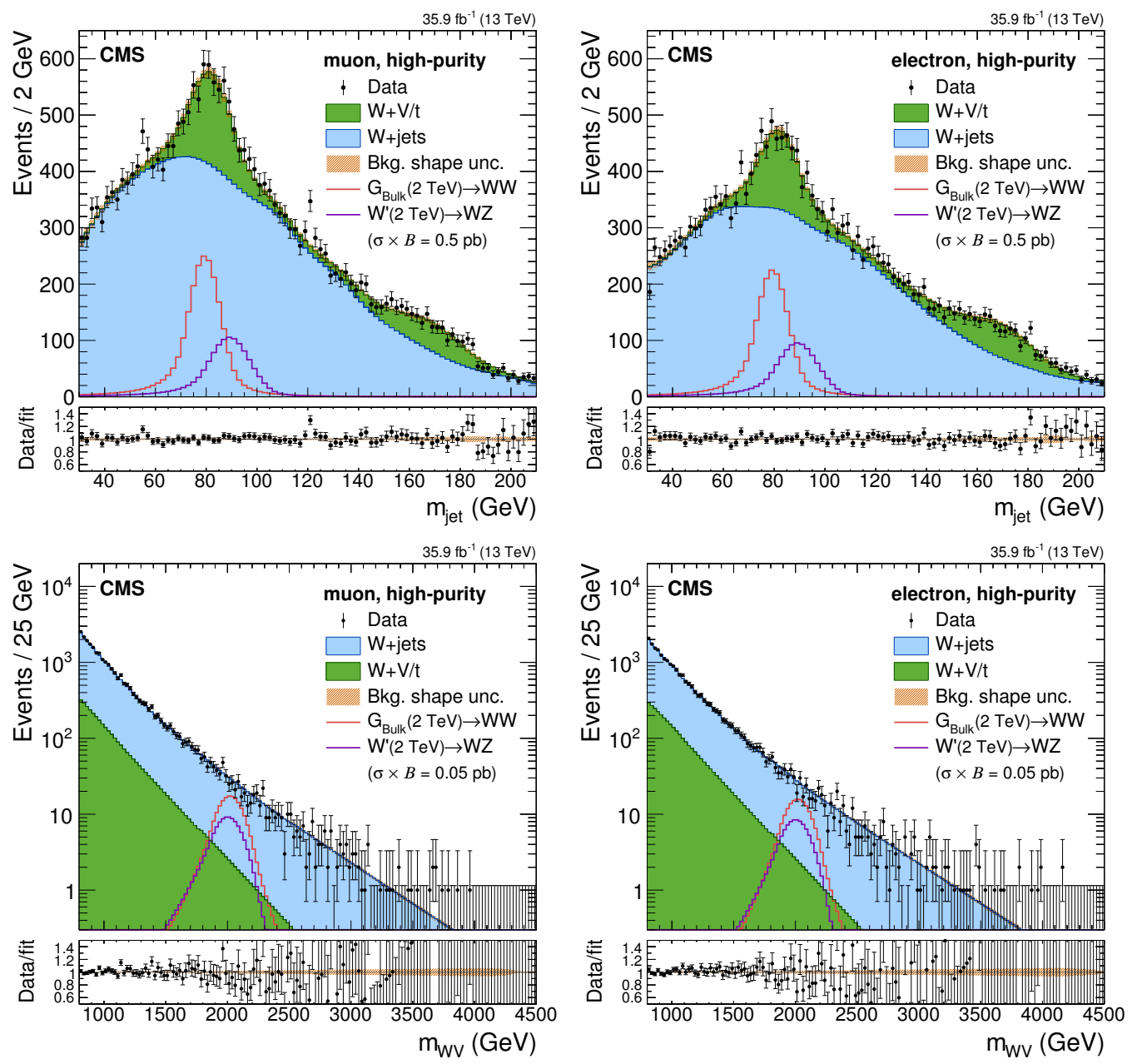

Figure 3. Comparison between the fit result and data distributions of $m_{\text {jet }}$ (upper) and $m_{\mathrm{WV}}$ (lower) in the muon HP (left) and electron HP (right) category. The background shape uncertainty is shown as a shaded band, and the statistical uncertainties of the data are shown as vertical bars. No events are observed with $m_{\mathrm{WV}}>4.5 \mathrm{TeV}$. Example signal distributions are overlaid, using an arbitrary normalization that is different in the upper and lower plots.

mass peak. After reducing the range, the observed and expected local significance was consistently lower, because of the loss in $t \bar{t}$ event count. Another test was to replace the 2D fit with a $1 \mathrm{D}$ binned fit on $m_{\mathrm{WV}}$ after introducing soft-drop jet mass windows similar to the $\alpha$ method. This test also yielded a background estimation compatible with the 2D fit result and a maximum local significance of 2.5 standard deviations.

The results are interpreted in terms of exclusion limits for the benchmark signal models described in section 3. We provide model-independent limits, which are not coupled to the relative normalizations of the benchmark models. We expect any model-dependent effects on the acceptance and selection efficiency to be covered by the PDF and scale uncertainties. Figure 6 shows the upper exclusion limits on the product of the resonance production cross section and the branching fraction to WW or WZ as a function of the resonance mass. The 

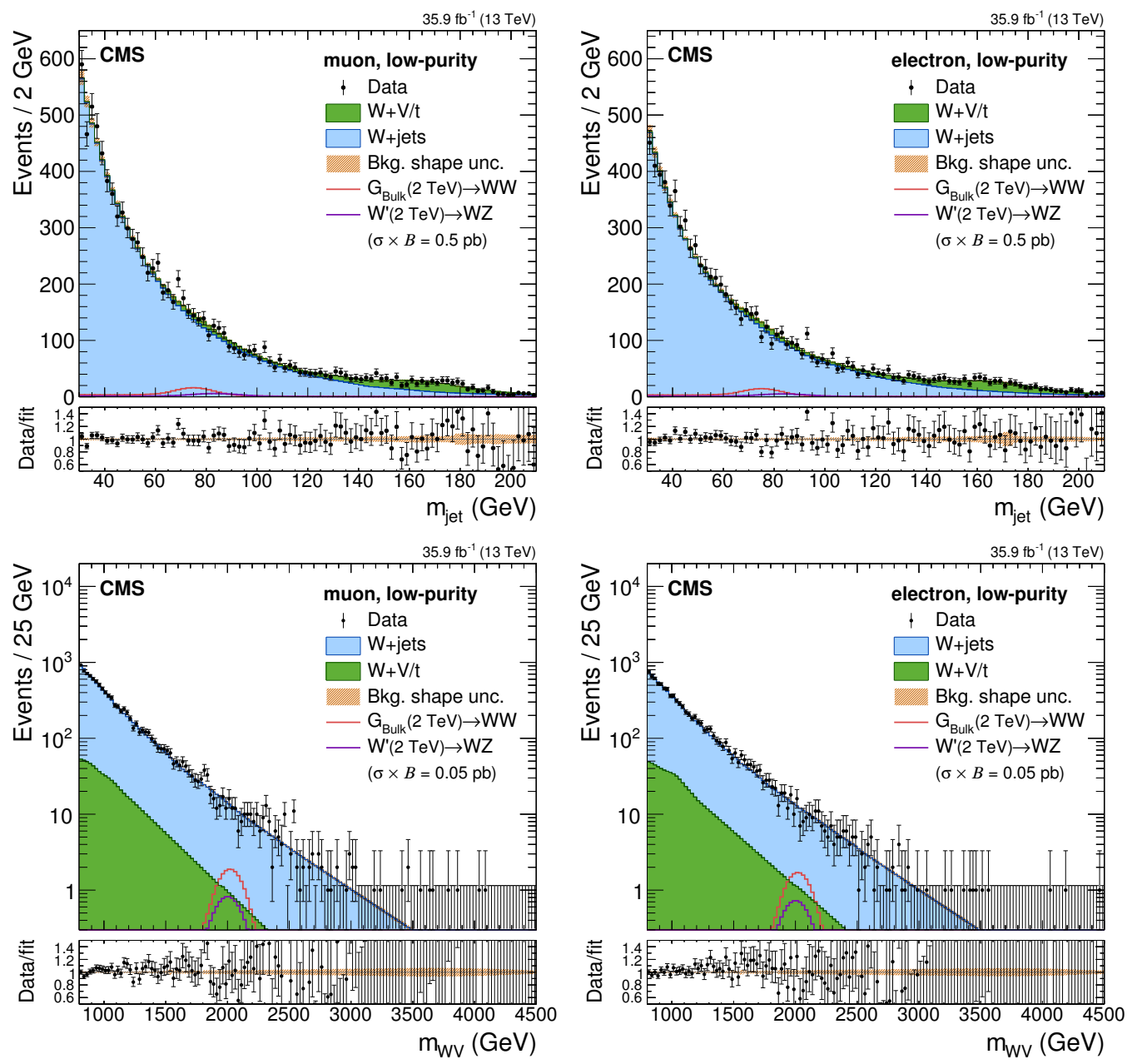

Figure 4. Comparison between the fit result and data distributions of $m_{\text {jet }}$ (upper) and $m_{\mathrm{WV}}$ (lower) in the muon LP (left) and electron LP (right) category. The background shape uncertainty is shown as a shaded band, and the statistical uncertainties of the data are shown as vertical bars. No events are observed with $m_{\mathrm{WV}}>4.5 \mathrm{TeV}$. Example signal distributions are overlaid, using an arbitrary normalization that is different in the upper and lower plots.

observed limits for the WW signal range from $29 \mathrm{fb}$ at $1.3 \mathrm{TeV}$ to $0.32 \mathrm{fb}$ at $4.4 \mathrm{TeV}$, while for the WZ signal they range from $84 \mathrm{fb}$ at $1.05 \mathrm{TeV}$ to $0.64 \mathrm{fb}$ at $4.4 \mathrm{TeV}$. By comparing these limits to the expected cross sections from the benchmark theoretical models, WW resonances lighter than $1.07 \mathrm{TeV}$ and $\mathrm{WZ}$ resonances lighter than $3.05 \mathrm{TeV}$ are excluded at $95 \%$ confidence level (CL), using the asymptotic approximation [86] of the $\mathrm{CL}_{\mathrm{s}}$ method [87, 88].

\section{Summary}

A search for a new heavy resonance decaying to a pair of vector bosons is performed in events with one muon or electron and a massive jet. Using the $N$-subjettiness ratio $\tau_{21}$, massive jets are tagged as highly energetic vector bosons $(\mathrm{V}=\mathrm{W}, \mathrm{Z})$ decaying to quark pairs. The soft-drop mass is used as an estimate of the V-jet mass. The lepton momentum 

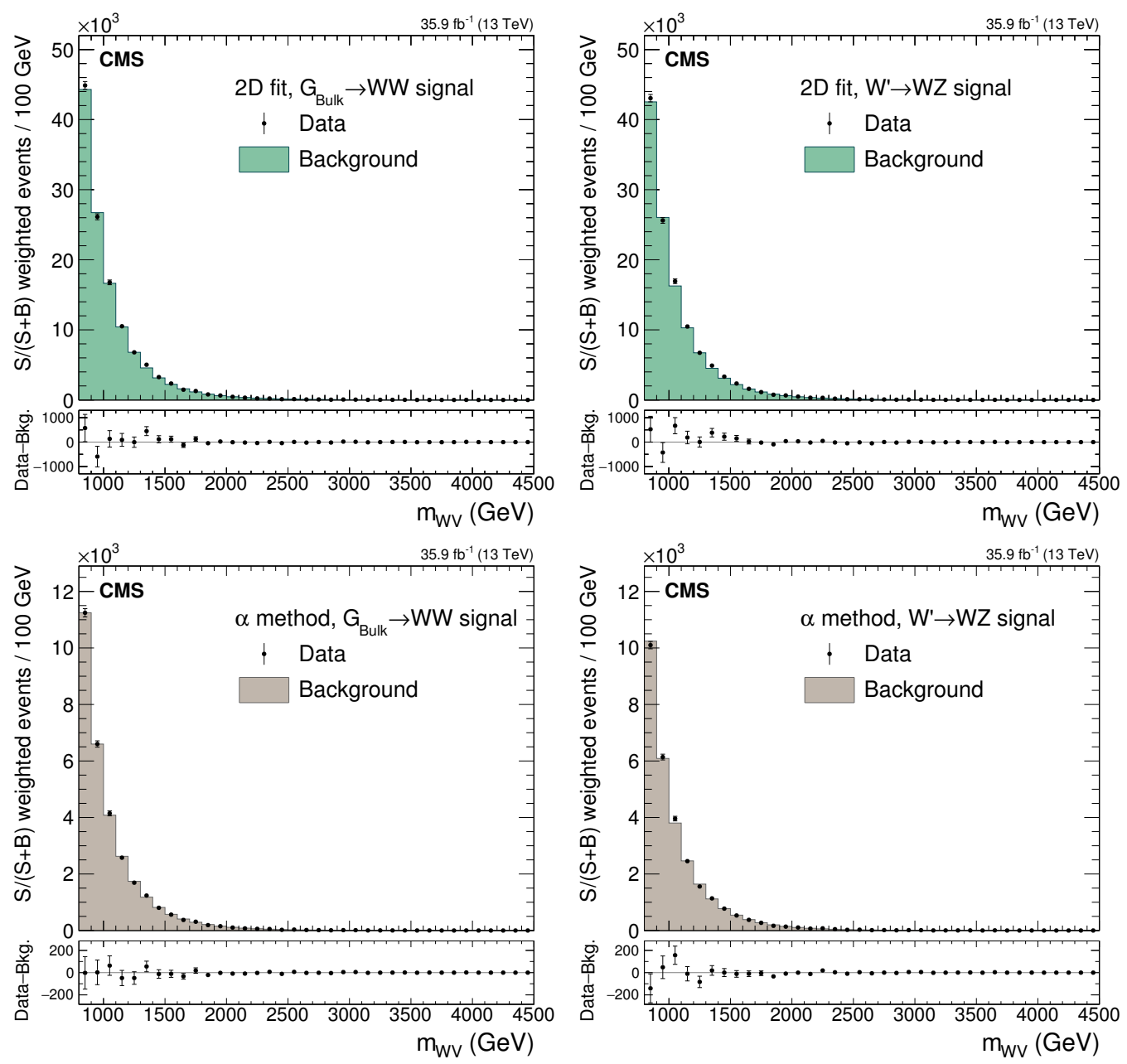

Figure 5. S/ $\mathrm{S}+\mathrm{B})$ event-weighted distributions of the resonance mass for the $\mathrm{G}_{\text {bulk }} \rightarrow \mathrm{WW}$ signal (left) and $\mathrm{W}^{\prime} \rightarrow \mathrm{WZ}$ signal (right) for the $2 \mathrm{D}$ fit (upper) and the $\alpha$ method (lower). The lower panels show the differences between the weighted data and the weighted fit results. The vertical bars correspond to the statistical uncertainties of the data.

and missing transverse momentum are used to reconstruct the momentum of the $\mathrm{W} \rightarrow \ell \nu$ boson candidate, constraining the invariant mass of the $\ell \nu$ pair to the $\mathrm{W}$ boson mass value. A novel signal extraction technique is introduced based on a simultaneous fit of the $\mathrm{V}$-jet mass and the diboson mass, and improves the search sensitivity compared to the method employed in previous versions of this analysis. No significant evidence of a new signal is found. The results are interpreted in terms of upper limits on the production cross section of new resonances decaying to WW and WZ final states. The observed limits for a WW resonance range from $29 \mathrm{fb}$ at $1.3 \mathrm{TeV}$ to $0.32 \mathrm{fb}$ at $4.4 \mathrm{TeV}$, while for a $\mathrm{WZ}$ resonance they range from $84 \mathrm{fb}$ at $1.05 \mathrm{TeV}$ to $0.64 \mathrm{fb}$ at $4.4 \mathrm{TeV}$. Comparing the excluded cross section values to the expectations from theoretical calculations, spin-2 bulk graviton resonances decaying to $\mathrm{WW}$ with mass smaller than $1.07 \mathrm{TeV}$ and $\mathrm{W}^{\prime} \rightarrow$ WZ resonances lighter than $3.05 \mathrm{TeV}$ are excluded at 95\% CL. 

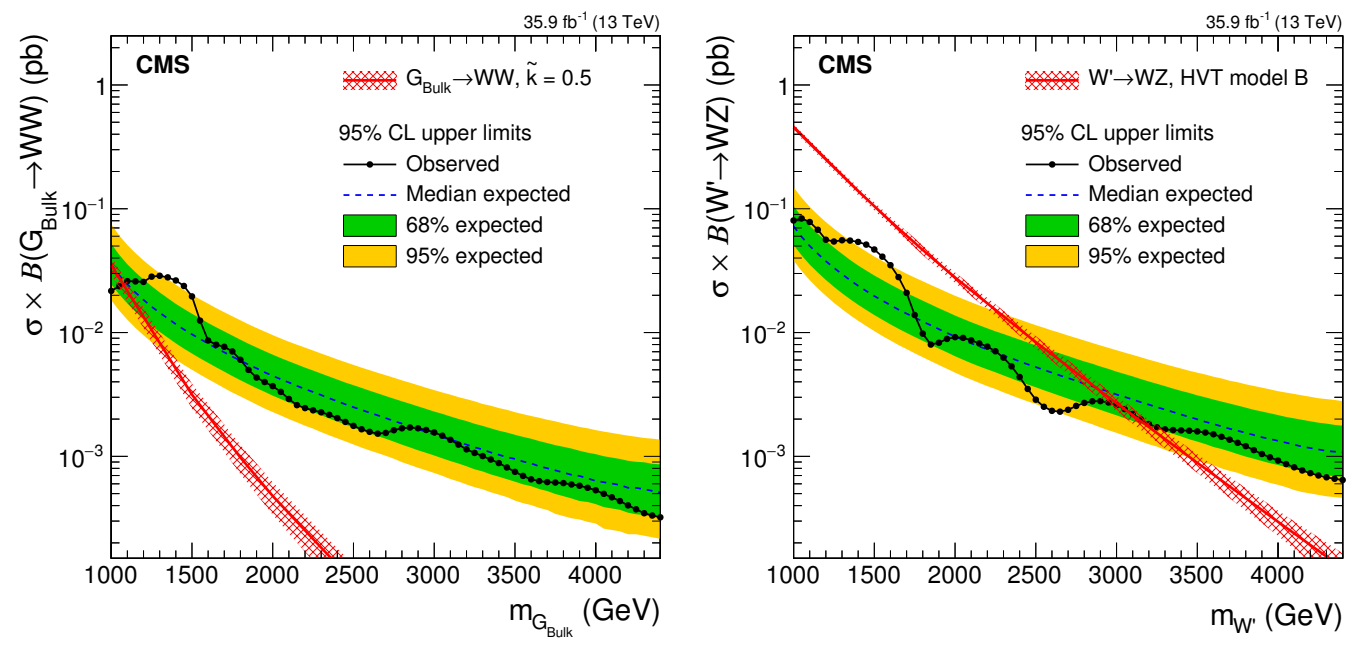

Figure 6. Exclusion limits on the product of the production cross section and the branching fraction for a new spin-2 resonance decaying to WW (left) and for a new spin-1 resonance decaying to WZ (right), as a function of the resonance mass hypothesis. Signal cross section uncertainties are shown as red cross-hatched bands.

\section{Acknowledgments}

We congratulate our colleagues in the CERN accelerator departments for the excellent performance of the LHC and thank the technical and administrative staffs at CERN and at other CMS institutes for their contributions to the success of the CMS effort. In addition, we gratefully acknowledge the computing centres and personnel of the Worldwide LHC Computing Grid for delivering so effectively the computing infrastructure essential to our analyses. Finally, we acknowledge the enduring support for the construction and operation of the LHC and the CMS detector provided by the following funding agencies: BMWFW and FWF (Austria); FNRS and FWO (Belgium); CNPq, CAPES, FAPERJ, and FAPESP (Brazil); MES (Bulgaria); CERN; CAS, MoST, and NSFC (China); COLCIENCIAS (Colombia); MSES and CSF (Croatia); RPF (Cyprus); SENESCYT (Ecuador); MoER, ERC IUT, and ERDF (Estonia); Academy of Finland, MEC, and HIP (Finland); CEA and CNRS/IN2P3 (France); BMBF, DFG, and HGF (Germany); GSRT (Greece); OTKA and NIH (Hungary); DAE and DST (India); IPM (Iran); SFI (Ireland); INFN (Italy); MSIP and NRF (Republic of Korea); LAS (Lithuania); MOE and UM (Malaysia); BUAP, CINVESTAV, CONACYT, LNS, SEP, and UASLP-FAI (Mexico); MBIE (New Zealand); PAEC (Pakistan); MSHE and NSC (Poland); FCT (Portugal); JINR (Dubna); MON, RosAtom, RAS, RFBR and RAEP (Russia); MESTD (Serbia); SEIDI, CPAN, PCTI and FEDER (Spain); Swiss Funding Agencies (Switzerland); MST (Taipei); ThEPCenter, IPST, STAR, and NSTDA (Thailand); TUBITAK and TAEK (Turkey); NASU and SFFR (Ukraine); STFC (United Kingdom); DOE and NSF (U.S.A.).

Individuals have received support from the Marie-Curie programme and the European Research Council and Horizon 2020 Grant, contract No. 675440 (European Union); the Leventis Foundation; the A. P. Sloan Foundation; the Alexander von Humboldt Foun- 
dation; the Belgian Federal Science Policy Office; the Fonds pour la Formation à la Recherche dans l'Industrie et dans l'Agriculture (FRIA-Belgium); the Agentschap voor Innovatie door Wetenschap en Technologie (IWT-Belgium); the F.R.S.-FNRS and FWO (Belgium) under the "Excellence of Science - EOS" - be.h project n. 30820817; the Ministry of Education, Youth and Sports (MEYS) of the Czech Republic; the Council of Science and Industrial Research, India; the HOMING PLUS programme of the Foundation for Polish Science, cofinanced from European Union, Regional Development Fund, the Mobility Plus programme of the Ministry of Science and Higher Education, the National Science Center (Poland), contracts Harmonia 2014/14/M/ST2/00428, Opus 2014/13/B/ST2/02543, 2014/15/B/ST2/03998, and 2015/19/B/ST2/02861, Sonata-bis 2012/07/E/ST2/01406; the National Priorities Research Program by Qatar National Research Fund; the Programa Estatal de Fomento de la Investigación Científica y Técnica de Excelencia María de Maeztu, grant MDM-2015-0509 and the Programa Severo Ochoa del Principado de Asturias; the Thalis and Aristeia programmes cofinanced by EU-ESF and the Greek NSRF; the Rachadapisek Sompot Fund for Postdoctoral Fellowship, Chulalongkorn University and the Chulalongkorn Academic into Its 2nd Century Project Advancement Project (Thailand); the Welch Foundation, contract C-1845; and the Weston Havens Foundation (U.S.A.).

Open Access. This article is distributed under the terms of the Creative Commons Attribution License (CC-BY 4.0), which permits any use, distribution and reproduction in any medium, provided the original author(s) and source are credited.

\section{References}

[1] ATLAS collaboration, Observation of a new particle in the search for the Standard Model Higgs boson with the ATLAS detector at the LHC, Phys. Lett. B 716 (2012) 1 [arXiv: 1207.7214] [INSPIRE].

[2] CMS collaboration, Observation of a new boson at a mass of $125 \mathrm{GeV}$ with the CMS experiment at the LHC, Phys. Lett. B 716 (2012) 30 [arXiv:1207.7235] [INSPIRE].

[3] CMS collaboration, Observation of a new boson with mass near $125 \mathrm{GeV}$ in pp collisions at $\sqrt{s}=7$ and $8 \mathrm{TeV}$, JHEP 06 (2013) 081 [arXiv:1303.4571] [INSPIRE].

[4] S.L. Glashow, Partial symmetries of weak interactions, Nucl. Phys. 22 (1961) 579 [InSPIRE].

[5] A. Salam and J.C. Ward, Electromagnetic and weak interactions, Phys. Lett. 13 (1964) 168 [INSPIRE].

[6] S. Weinberg, A model of leptons, Phys. Rev. Lett. 19 (1967) 1264 [inSPIRE].

[7] L. Susskind, Dynamics of spontaneous symmetry breaking in the Weinberg-Salam theory, Phys. Rev. D 20 (1979) 2619 [INSPIRE].

[8] G. 't Hooft, Naturalness, chiral symmetry, and spontaneous chiral symmetry breaking, NATO Sci. Ser. B 59 (1980) 135 [INSPIRE].

[9] R. Barbieri and G.F. Giudice, Upper bounds on supersymmetric particle masses, Nucl. Phys. B 306 (1988) 63 [INSPIRE]. 
[10] G.F. Giudice, Naturally speaking: the naturalness criterion and physics at the LHC, arXiv:0801.2562 [INSPIRE].

[11] L. Randall and R. Sundrum, A large mass hierarchy from a small extra dimension, Phys. Rev. Lett. 83 (1999) 3370 [hep-ph/9905221] [INSPIRE].

[12] L. Randall and R. Sundrum, An alternative to compactification, Phys. Rev. Lett. 83 (1999) 4690 [hep-th/9906064] [INSPIRE].

[13] K. Agashe, H. Davoudiasl, G. Perez and A. Soni, Warped gravitons at the LHC and beyond, Phys. Rev. D 76 (2007) 036006 [hep-ph/0701186] [INSPIRE].

[14] A.L. Fitzpatrick, J. Kaplan, L. Randall and L.-T. Wang, Searching for the Kaluza-Klein graviton in bulk RS models, JHEP 09 (2007) 013 [hep-ph/0701150] [INSPIRE].

[15] K. Agashe, R. Contino and A. Pomarol, The minimal composite Higgs model, Nucl. Phys. B 719 (2005) 165 [hep-ph/0412089] [INSPIRE].

[16] R. Contino, Y. Nomura and A. Pomarol, Higgs as a holographic pseudo-Goldstone boson, Nucl. Phys. B 671 (2003) 148 [hep-ph/0306259] [INSPIRE].

[17] R. Contino, T. Kramer, M. Son and R. Sundrum, Warped/composite phenomenology simplified, JHEP 05 (2007) 074 [hep-ph/0612180] [INSPIRE].

[18] K. Lane and L. Pritchett, The light composite Higgs boson in strong extended technicolor, JHEP 06 (2017) 140 [arXiv: 1604.07085] [INSPIRE].

[19] CMS collaboration, Search for massive resonances in dijet systems containing jets tagged as $W$ or $Z$ boson decays in pp collisions at $\sqrt{s}=8$ TeV, JHEP 08 (2014) 173 [arXiv: 1405.1994] [INSPIRE].

[20] CMS collaboration, Search for massive resonances decaying into pairs of boosted bosons in semi-leptonic final states at $\sqrt{s}=8 \mathrm{TeV}$, JHEP 08 (2014) 174 [arXiv:1405.3447] [INSPIRE].

[21] CMS collaboration, Search for a massive resonance decaying into a Higgs boson and a $W$ or $Z$ boson in hadronic final states in proton-proton collisions at $\sqrt{s}=8 \mathrm{TeV}$, JHEP 02 (2016) 145 [arXiv: 1506.01443] [INSPIRE].

[22] CMS collaboration, Search for narrow high-mass resonances in proton-proton collisions at $\sqrt{s}=8 \mathrm{TeV}$ decaying to a $Z$ and a Higgs boson, Phys. Lett. B 748 (2015) 255 [arXiv: 1502.04994] [INSPIRE].

[23] CMS collaboration, Search for massive WH resonances decaying into the $\ell \nu b \bar{b}$ final state at $\sqrt{s}=8 \mathrm{TeV}$, Eur. Phys. J. C 76 (2016) 237 [arXiv:1601.06431] [InSPIRE].

[24] CMS collaboration, Search for heavy resonances decaying to two Higgs bosons in final states containing four b quarks, Eur. Phys. J. C 76 (2016) 371 [arXiv:1602.08762] [INSPIRE].

[25] CMS collaboration, Search for massive resonances decaying into $W W, W Z$ or $Z Z$ bosons in proton-proton collisions at $\sqrt{s}=13 \mathrm{TeV}$, JHEP 03 (2017) 162 [arXiv:1612.09159] [INSPIRE].

[26] CMS collaboration, Search for heavy resonances decaying into a vector boson and a Higgs boson in final states with charged leptons, neutrinos and b quarks, Phys. Lett. B 768 (2017) 137 [arXiv: 1610.08066] [INSPIRE].

[27] ATLAS collaboration, Combination of searches for $W W, W Z$ and $Z Z$ resonances in $p p$ collisions at $\sqrt{s}=8 \mathrm{TeV}$ with the ATLAS detector, Phys. Lett. B 755 (2016) 285 [arXiv: 1512.05099] [INSPIRE]. 
[28] ATLAS collaboration, Search for high-mass diboson resonances with boson-tagged jets in proton-proton collisions at $\sqrt{s}=8 \mathrm{TeV}$ with the ATLAS detector, JHEP 12 (2015) 055 [arXiv: 1506.00962] [INSPIRE].

[29] ATLAS collaboration, Search for a new resonance decaying to a $W$ or $Z$ boson and a Higgs boson in the $\ell \ell / \ell \nu / \nu \nu+b \bar{b}$ final states with the ATLAS detector, Eur. Phys. J. C 75 (2015) 263 [arXiv: 1503.08089] [INSPIRE].

[30] ATLAS collaboration, Search for production of $W W / W Z$ resonances decaying to a lepton, neutrino and jets in pp collisions at $\sqrt{s}=8 \mathrm{TeV}$ with the ATLAS detector, Eur. Phys. J. C 75 (2015) 209 [Erratum ibid. C 75 (2015) 370] [arXiv: 1503.04677] [INSPIRE].

[31] ATLAS collaboration, Search for resonant diboson production in the $\ell \ell q \bar{q}$ final state in $p p$ collisions at $\sqrt{s}=8 \mathrm{TeV}$ with the ATLAS detector, Eur. Phys. J. C 75 (2015) 69 [arXiv: 1409.6190] [INSPIRE].

[32] ATLAS collaboration, Search for new resonances decaying to a $W$ or $Z$ boson and a Higgs boson in the $\ell^{+} \ell^{-} b \bar{b}, \ell \nu b \bar{b}$ and $\nu \bar{\nu} b \bar{b}$ channels with pp collisions at $\sqrt{s}=13 \mathrm{TeV}$ with the ATLAS detector, Phys. Lett. B 765 (2017) 32 [arXiv: 1607.05621] [INSPIRE].

[33] ATLAS collaboration, Searches for heavy diboson resonances in $p p$ collisions at $\sqrt{s}=13 \mathrm{TeV}$ with the ATLAS detector, JHEP 09 (2016) 173 [arXiv:1606. 04833] [INSPIRE].

[34] D. Pappadopulo, A. Thamm, R. Torre and A. Wulzer, Heavy vector triplets: bridging theory and data, JHEP 09 (2014) 060 [arXiv:1402.4431] [INSPIRE].

[35] ATLAS collaboration, Search for $W W / W Z$ resonance production in $\ell \nu q q$ final states in $p p$ collisions at $\sqrt{s}=13 \mathrm{TeV}$ with the ATLAS detector, JHEP 03 (2018) 042 [arXiv: 1710.07235] [INSPIRE].

[36] CMS collaboration, The CMS experiment at the CERN LHC, 2008 JINST 3 S08004 [INSPIRE].

[37] A. Oliveira, Gravity particles from warped extra dimensions, predictions for LHC, arXiv: 1404.0102 [INSPIRE].

[38] B. Bellazzini, C. Csáki and J. Serra, Composite higgses, Eur. Phys. J. C 74 (2014) 2766 [arXiv: 1401.2457] [INSPIRE].

[39] R. Contino, D. Marzocca, D. Pappadopulo and R. Rattazzi, On the effect of resonances in composite Higgs phenomenology, JHEP 10 (2011) 081 [arXiv:1109.1570] [INSPIRE].

[40] D. Marzocca, M. Serone and J. Shu, General composite Higgs models, JHEP 08 (2012) 013 [arXiv:1205.0770] [INSPIRE].

[41] D. Greco and D. Liu, Hunting composite vector resonances at the LHC: naturalness facing data, JHEP 12 (2014) 126 [arXiv:1410.2883] [InSPIRE].

[42] J. Alwall et al., The automated computation of tree-level and next-to-leading order differential cross sections and their matching to parton shower simulations, JHEP 07 (2014) 079 [arXiv: 1405.0301] [INSPIRE].

[43] Y. Li and F. Petriello, Combining QCD and electroweak corrections to dilepton production in FEWZ, Phys. Rev. D 86 (2012) 094034 [arXiv:1208.5967] [InSPIRE].

[44] S. Kallweit, J.M. Lindert, P. Maierhofer, S. Pozzorini and M. Schönherr, NLO QCD + EW predictions for $V+$ jets including off-shell vector-boson decays and multijet merging, JHEP 04 (2016) 021 [arXiv:1511.08692] [INSPIRE]. 
[45] P. Nason, A new method for combining NLO QCD with shower Monte Carlo algorithms, JHEP 11 (2004) 040 [hep-ph/0409146] [INSPIRE].

[46] S. Frixione, P. Nason and C. Oleari, Matching NLO QCD computations with parton shower simulations: the POWHEG method, JHEP 11 (2007) 070 [arXiv:0709.2092] [INSPIRE].

[47] S. Alioli, P. Nason, C. Oleari and E. Re, A general framework for implementing NLO calculations in shower Monte Carlo programs: the POWHEG BOX, JHEP 06 (2010) 043 [arXiv: 1002.2581] [INSPIRE].

[48] S. Alioli, P. Nason, C. Oleari and E. Re, NLO single-top production matched with shower in POWHEG: s- and t-channel contributions, JHEP 09 (2009) 111 [Erratum ibid. 02 (2010) 011] [arXiv:0907.4076] [inSPIRE].

[49] E. Re, Single-top Wt-channel production matched with parton showers using the POWHEG method, Eur. Phys. J. C 71 (2011) 1547 [arXiv:1009.2450] [INSPIRE].

[50] S. Alioli, S.-O. Moch and P. Uwer, Hadronic top-quark pair-production with one jet and parton showering, JHEP 01 (2012) 137 [arXiv:1110.5251] [INSPIRE].

[51] M. Czakon and A. Mitov, Top++: a program for the calculation of the top-pair cross-section at hadron colliders, Comput. Phys. Commun. 185 (2014) 2930 [arXiv:1112.5675] [InSPIRE].

[52] R. Frederix and S. Frixione, Merging meets matching in MC@NLO, JHEP 12 (2012) 061 [arXiv:1209.6215] [INSPIRE].

[53] J.M. Campbell, R.K. Ellis and C. Williams, Vector boson pair production at the LHC, JHEP 07 (2011) 018 [arXiv: 1105.0020] [INSPIRE].

[54] J.M. Campbell and R.K. Ellis, Top-quark processes at NLO in production and decay, J. Phys. G 42 (2015) 015005 [arXiv: 1204.1513] [INSPIRE].

[55] J.M. Campbell, R.K. Ellis and F. Tramontano, Single top production and decay at next-to-leading order, Phys. Rev. D 70 (2004) 094012 [hep-ph/0408158] [INSPIRE].

[56] T. Sjöstrand, S. Mrenna and P.Z. Skands, PYTHIA 6.4 physics and manual, JHEP 05 (2006) 026 [hep-ph/0603175] [inSPIRE].

[57] T. Sjöstrand, S. Mrenna and P.Z. Skands, A brief introduction to PYTHIA 8.1, Comput. Phys. Commun. 178 (2008) 852 [arXiv:0710.3820] [INSPIRE].

[58] P. Skands, S. Carrazza and J. Rojo, Tuning PYTHIA 8.1: the Monash 2013 tune, Eur. Phys. J. C 74 (2014) 3024 [arXiv: 1404.5630] [InSPIRE].

[59] CMS collaboration, Event generator tunes obtained from underlying event and multiparton scattering measurements, Eur. Phys. J. C 76 (2016) 155 [arXiv:1512.00815] [inSPIRE].

[60] R.D. Ball et al., Impact of heavy quark masses on parton distributions and LHC phenomenology, Nucl. Phys. B 849 (2011) 296 [arXiv:1101.1300] [INSPIRE].

[61] GEANT4 collaboration, S. Agostinelli et al., GEANT4: a simulation toolkit, Nucl. Instrum. Meth. A 506 (2003) 250 [INSPIRE].

[62] CMS collaboration, Measurements of inclusive $W$ and $Z$ cross sections in pp collisions at $\sqrt{s}=7 \mathrm{TeV}$, JHEP 01 (2011) 080 [arXiv:1012.2466] [INSPIRE].

[63] CMS collaboration, Identification of heavy-flavour jets with the CMS detector in $p p$ collisions at $13 \mathrm{TeV}$, submitted to JINST (2017) [arXiv:1712.07158] [INSPIRE]. 
[64] CMS collaboration, Particle-flow reconstruction and global event description with the CMS detector, 2017 JINST 12 P10003 [arXiv:1706.04965] [INSPIRE].

[65] CMS collaboration, Description and performance of track and primary-vertex reconstruction with the CMS tracker, 2014 JINST 9 P10009 [arXiv:1405.6569] [INSPIRE].

[66] M. Cacciari, G.P. Salam and G. Soyez, The anti- $k_{t}$ jet clustering algorithm, JHEP 04 (2008) 063 [arXiv:0802.1189] [INSPIRE].

[67] M. Cacciari, G.P. Salam and G. Soyez, FastJet user manual, Eur. Phys. J. C 72 (2012) 1896 [arXiv:1111.6097] [INSPIRE].

[68] CMS collaboration, The CMS trigger system, 2017 JINST 12 P01020 [arXiv:1609.02366] [INSPIRE].

[69] CMS collaboration, Performance of electron reconstruction and selection with the CMS detector in proton-proton collisions at $\sqrt{s}=8 \mathrm{TeV}, 2015$ JINST $10 \mathrm{P} 06005$ [arXiv: 1502.02701] [INSPIRE].

[70] CMS collaboration, Performance of CMS muon reconstruction in pp collision events at $\sqrt{s}=7$ TeV, 2012 JINST 7 P10002 [arXiv: 1206.4071] [INSPIRE].

[71] CMS collaboration, Jet energy scale and resolution in the CMS experiment in pp collisions at $8 \mathrm{TeV}, 2017$ JINST 12 P02014 [arXiv: 1607.03663] [INSPIRE].

[72] D. Bertolini, P. Harris, M. Low and N. Tran, Pileup per particle identification, JHEP 10 (2014) 059 [arXiv:1407.6013] [INSPIRE].

[73] CMS collaboration, Jet algorithms performance in 13 TeV data, CMS-PAS-JME-16-003, CERN, Geneva Switzerland, (2016).

[74] CMS collaboration, Performance of missing energy reconstruction in 13 TeV pp collision data using the CMS detector, CMS-PAS-JME-16-004, CERN, Geneva Switzerland, (2016).

[75] J. Thaler and K. Van Tilburg, Identifying boosted objects with N-subjettiness, JHEP 03 (2011) 015 [arXiv: 1011.2268] [INSPIRE].

[76] M. Dasgupta, A. Fregoso, S. Marzani and G.P. Salam, Towards an understanding of jet substructure, JHEP 09 (2013) 029 [arXiv: 1307.0007] [INSPIRE].

[77] J.M. Butterworth, A.R. Davison, M. Rubin and G.P. Salam, Jet substructure as a new Higgs search channel at the LHC, Phys. Rev. Lett. 100 (2008) 242001 [arXiv:0802.2470] [INSPIRE].

[78] A.J. Larkoski, S. Marzani, G. Soyez and J. Thaler, Soft drop, JHEP 05 (2014) 146 [arXiv: 1402.2657] [INSPIRE].

[79] M.J. Oreglia, A study of the reactions $\psi^{\prime} \rightarrow \gamma \gamma \psi$, Ph.D. thesis, SLAC report SLAC-R-236, Stanford University, U.S.A., (1980) [INSPIRE].

[80] J. Gaiser, Charmonium spectroscopy from radiative decays of the $J / \psi$ and $\psi^{\prime}$, Ph.D. thesis, SLAC report SLAC-R-255, Stanford University, U.S.A., (1982) [INSPIRE].

[81] K.S. Cranmer, Kernel estimation in high-energy physics, Comput. Phys. Commun. 136 (2001) 198 [hep-ex/0011057] [INSPIRE].

[82] CMS collaboration, CMS luminosity measurements for the 2016 data taking period, CMS-PAS-LUM-17-001, CERN, Geneva Switzerland, (2017). 
[83] M. Cacciari, S. Frixione, M.L. Mangano, P. Nason and G. Ridolfi, The t $\bar{t}$ cross-section at $1.8 \mathrm{TeV}$ and $1.96 \mathrm{TeV}$ : a study of the systematics due to parton densities and scale dependence, JHEP 04 (2004) 068 [hep-ph/0303085] [INSPIRE].

[84] S. Catani, D. de Florian, M. Grazzini and P. Nason, Soft gluon resummation for Higgs boson production at hadron colliders, JHEP 07 (2003) 028 [hep-ph/0306211] [INSPIRE].

[85] S. Baker and R.D. Cousins, Clarification of the use of $\chi^{2}$ and likelihood functions in fits to histograms, Nucl. Instrum. Meth. 221 (1984) 437 [INSPIRE].

[86] G. Cowan, K. Cranmer, E. Gross and O. Vitells, Asymptotic formulae for likelihood-based tests of new physics, Eur. Phys. J. C 71 (2011) 1554 [Erratum ibid. C 73 (2013) 2501] [arXiv: 1007.1727] [INSPIRE].

[87] T. Junk, Confidence level computation for combining searches with small statistics, Nucl. Instrum. Meth. A 434 (1999) 435 [hep-ex/9902006] [INSPIRE].

[88] A.L. Read, Presentation of search results: the $C L_{s}$ technique, J. Phys. G 28 (2002) 2693 [INSPIRE]. 


\section{The CMS collaboration}

\section{Yerevan Physics Institute, Yerevan, Armenia}

A.M. Sirunyan, A. Tumasyan

\section{Institut für Hochenergiephysik, Wien, Austria}

W. Adam, F. Ambrogi, E. Asilar, T. Bergauer, J. Brandstetter, E. Brondolin, M. Dragicevic, J. Erö, A. Escalante Del Valle, M. Flechl, M. Friedl, R. Frühwirth ${ }^{1}$, V.M. Ghete, J. Grossmann, J. Hrubec, M. Jeitler ${ }^{1}$, A. König, N. Krammer, I. Krätschmer, D. Liko, T. Madlener, I. Mikulec, E. Pree, N. Rad, H. Rohringer, J. Schieck ${ }^{1}$, R. Schöfbeck, M. Spanring, D. Spitzbart, A. Taurok, W. Waltenberger, J. Wittmann, C.-E. Wulz ${ }^{1}$, M. Zarucki

\section{Institute for Nuclear Problems, Minsk, Belarus}

V. Chekhovsky, V. Mossolov, J. Suarez Gonzalez

\section{Universiteit Antwerpen, Antwerpen, Belgium}

E.A. De Wolf, D. Di Croce, X. Janssen, J. Lauwers, M. Pieters, M. Van De Klundert, H. Van Haevermaet, P. Van Mechelen, N. Van Remortel

\section{Vrije Universiteit Brussel, Brussel, Belgium}

S. Abu Zeid, F. Blekman, J. D'Hondt, I. De Bruyn, J. De Clercq, K. Deroover, G. Flouris, D. Lontkovskyi, S. Lowette, I. Marchesini, S. Moortgat, L. Moreels, Q. Python, K. Skovpen, S. Tavernier, W. Van Doninck, P. Van Mulders, I. Van Parijs

\section{Université Libre de Bruxelles, Bruxelles, Belgium}

D. Beghin, B. Bilin, H. Brun, B. Clerbaux, G. De Lentdecker, H. Delannoy, B. Dorney, G. Fasanella, L. Favart, R. Goldouzian, A. Grebenyuk, A.K. Kalsi, T. Lenzi, J. Luetic, T. Seva, E. Starling, C. Vander Velde, P. Vanlaer, D. Vannerom, R. Yonamine

\section{Ghent University, Ghent, Belgium}

T. Cornelis, D. Dobur, A. Fagot, M. Gul, I. Khvastunov², D. Poyraz, C. Roskas, D. Trocino, M. Tytgat, W. Verbeke, B. Vermassen, M. Vit, N. Zaganidis

\section{Université Catholique de Louvain, Louvain-la-Neuve, Belgium}

H. Bakhshiansohi, O. Bondu, S. Brochet, G. Bruno, C. Caputo, A. Caudron, P. David, S. De Visscher, C. Delaere, M. Delcourt, B. Francois, A. Giammanco, G. Krintiras, V. Lemaitre, A. Magitteri, A. Mertens, M. Musich, K. Piotrzkowski, L. Quertenmont, A. Saggio, M. Vidal Marono, S. Wertz, J. Zobec

\section{Centro Brasileiro de Pesquisas Fisicas, Rio de Janeiro, Brazil}

W.L. Aldá Júnior, F.L. Alves, G.A. Alves, L. Brito, G. Correia Silva, C. Hensel, A. Moraes, M.E. Pol, P. Rebello Teles

Universidade do Estado do Rio de Janeiro, Rio de Janeiro, Brazil

E. Belchior Batista Das Chagas, W. Carvalho, J. Chinellato ${ }^{3}$, E. Coelho, E.M. Da Costa, G.G. Da Silveira ${ }^{4}$, D. De Jesus Damiao, S. Fonseca De Souza, L.M. Huertas Guativa, H. Malbouisson, M. Medina Jaime ${ }^{5}$, M. Melo De Almeida, C. Mora Herrera, 
L. Mundim, H. Nogima, L.J. Sanchez Rosas, A. Santoro, A. Sznajder, M. Thiel, E.J. Tonelli Manganote $^{3}$, F. Torres Da Silva De Araujo, A. Vilela Pereira

Universidade Estadual Paulista $^{a}$, Universidade Federal do ABC ${ }^{b}$, São Paulo, Brazil

S. Ahuja ${ }^{a}$, C.A. Bernardes ${ }^{a}$, L. Calligaris ${ }^{a}$, T.R. Fernandez Perez Tomei ${ }^{a}$, E.M. Gregores $^{b}$, P.G. Mercadante ${ }^{b}$, S.F. Novaes ${ }^{a}$, Sandra S. Padula ${ }^{a}$, D. Romero $\operatorname{Abad}^{b}$, J.C. Ruiz Vargas ${ }^{a}$

Institute for Nuclear Research and Nuclear Energy, Bulgarian Academy of Sciences, Sofia, Bulgaria

A. Aleksandrov, R. Hadjiiska, P. Iaydjiev, A. Marinov, M. Misheva, M. Rodozov, M. Shopova, G. Sultanov

University of Sofia, Sofia, Bulgaria

A. Dimitrov, L. Litov, B. Pavlov, P. Petkov

Beihang University, Beijing, China

W. Fang ${ }^{6}$, X. Gao ${ }^{6}$, L. Yuan

Institute of High Energy Physics, Beijing, China

M. Ahmad, J.G. Bian, G.M. Chen, H.S. Chen, M. Chen, Y. Chen, C.H. Jiang, D. Leggat, H. Liao, Z. Liu, F. Romeo, S.M. Shaheen, A. Spiezia, J. Tao, C. Wang, Z. Wang, E. Yazgan, H. Zhang, J. Zhao

State Key Laboratory of Nuclear Physics and Technology, Peking University, Beijing, China

Y. Ban, G. Chen, H. Huang, J. Li, Q. Li, S. Liu, Y. Mao, S.J. Qian, D. Wang, Z. Xu

Tsinghua University, Beijing, China

Y. Wang

Universidad de Los Andes, Bogota, Colombia

C. Avila, A. Cabrera, C.A. Carrillo Montoya, L.F. Chaparro Sierra, C. Florez, C.F. González Hernández, M.A. Segura Delgado

University of Split, Faculty of Electrical Engineering, Mechanical Engineering and Naval Architecture, Split, Croatia

B. Courbon, N. Godinovic, D. Lelas, I. Puljak, P.M. Ribeiro Cipriano, T. Sculac

University of Split, Faculty of Science, Split, Croatia

Z. Antunovic, M. Kovac

Institute Rudjer Boskovic, Zagreb, Croatia

V. Brigljevic, D. Ferencek, K. Kadija, B. Mesic, A. Starodumov ${ }^{7}$, T. Susa

University of Cyprus, Nicosia, Cyprus

M.W. Ather, A. Attikis, G. Mavromanolakis, J. Mousa, C. Nicolaou, F. Ptochos, P.A. Razis, H. Rykaczewski

Charles University, Prague, Czech Republic

M. Finger ${ }^{8}$, M. Finger Jr. ${ }^{8}$ 
Universidad San Francisco de Quito, Quito, Ecuador

E. Carrera Jarrin

Academy of Scientific Research and Technology of the Arab Republic of Egypt, Egyptian Network of High Energy Physics, Cairo, Egypt

M.A. Mahmoud ${ }^{9,10}$, Y. Mohammed ${ }^{9}$, E. Salama ${ }^{10,11}$

National Institute of Chemical Physics and Biophysics, Tallinn, Estonia

S. Bhowmik, R.K. Dewanjee, M. Kadastik, L. Perrini, M. Raidal, C. Veelken

Department of Physics, University of Helsinki, Helsinki, Finland

P. Eerola, H. Kirschenmann, J. Pekkanen, M. Voutilainen

Helsinki Institute of Physics, Helsinki, Finland

J. Havukainen, J.K. Heikkilä, T. Järvinen, V. Karimäki, R. Kinnunen, T. Lampén,

K. Lassila-Perini, S. Laurila, S. Lehti, T. Lindén, P. Luukka, T. Mäenpää, H. Siikonen,

E. Tuominen, J. Tuominiemi

Lappeenranta University of Technology, Lappeenranta, Finland

T. Tuuva

IRFU, CEA, Université Paris-Saclay, Gif-sur-Yvette, France

M. Besancon, F. Couderc, M. Dejardin, D. Denegri, J.L. Faure, F. Ferri, S. Ganjour, S. Ghosh, A. Givernaud, P. Gras, G. Hamel de Monchenault, P. Jarry, C. Leloup, E. Locci, M. Machet, J. Malcles, G. Negro, J. Rander, A. Rosowsky, M.Ö. Sahin, M. Titov

Laboratoire Leprince-Ringuet, Ecole polytechnique, CNRS/IN2P3, Université Paris-Saclay, Palaiseau, France

A. Abdulsalam ${ }^{12}$, C. Amendola, I. Antropov, S. Baffioni, F. Beaudette, P. Busson,

L. Cadamuro, C. Charlot, R. Granier de Cassagnac, M. Jo, I. Kucher, S. Lisniak,

A. Lobanov, J. Martin Blanco, M. Nguyen, C. Ochando, G. Ortona, P. Paganini, P. Pigard,

R. Salerno, J.B. Sauvan, Y. Sirois, A.G. Stahl Leiton, Y. Yilmaz, A. Zabi, A. Zghiche

Université de Strasbourg, CNRS, IPHC UMR 7178, F-67000 Strasbourg, France

J.-L. Agram ${ }^{13}$, J. Andrea, D. Bloch, J.-M. Brom, M. Buttignol, E.C. Chabert, C. Collard, E. Conte ${ }^{13}$, X. Coubez, F. Drouhin ${ }^{13}$, J.-C. Fontaine ${ }^{13}$, D. Gelé, U. Goerlach, M. Jansová, P. Juillot, A.-C. Le Bihan, N. Tonon, P. Van Hove

Centre de Calcul de l'Institut National de Physique Nucleaire et de Physique des Particules, CNRS/IN2P3, Villeurbanne, France

S. Gadrat

Université de Lyon, Université Claude Bernard Lyon 1, CNRS-IN2P3, Institut de Physique Nucléaire de Lyon, Villeurbanne, France

S. Beauceron, C. Bernet, G. Boudoul, N. Chanon, R. Chierici, D. Contardo, P. Depasse, H. El Mamouni, J. Fay, L. Finco, S. Gascon, M. Gouzevitch, G. Grenier, B. Ille, F. Lagarde, I.B. Laktineh, H. Lattaud, M. Lethuillier, L. Mirabito, A.L. Pequegnot, S. Perries, A. Popov ${ }^{14}$, V. Sordini, M. Vander Donckt, S. Viret, S. Zhang 
Georgian Technical University, Tbilisi, Georgia

T. Toriashvili ${ }^{15}$

Tbilisi State University, Tbilisi, Georgia

Z. Tsamalaidze ${ }^{8}$

RWTH Aachen University, I. Physikalisches Institut, Aachen, Germany

C. Autermann, L. Feld, M.K. Kiesel, K. Klein, M. Lipinski, M. Preuten, C. Schomakers, J. Schulz, M. Teroerde, B. Wittmer, V. Zhukov ${ }^{14}$

RWTH Aachen University, III. Physikalisches Institut A, Aachen, Germany A. Albert, D. Duchardt, M. Endres, M. Erdmann, S. Erdweg, T. Esch, R. Fischer, A. Güth, T. Hebbeker, C. Heidemann, K. Hoepfner, S. Knutzen, M. Merschmeyer, A. Meyer, P. Millet, S. Mukherjee, T. Pook, M. Radziej, H. Reithler, M. Rieger, F. Scheuch, D. Teyssier, S. Thüer

RWTH Aachen University, III. Physikalisches Institut B, Aachen, Germany

G. Flügge, B. Kargoll, T. Kress, A. Künsken, T. Müller, A. Nehrkorn, A. Nowack, C. Pistone, O. Pooth, A. Stahl ${ }^{16}$

\section{Deutsches Elektronen-Synchrotron, Hamburg, Germany}

M. Aldaya Martin, T. Arndt, C. Asawatangtrakuldee, K. Beernaert, O. Behnke, U. Behrens, A. Bermúdez Martínez, A.A. Bin Anuar, K. Borras ${ }^{17}$, V. Botta, A. Campbell, P. Connor, C. Contreras-Campana, F. Costanza, V. Danilov, A. De Wit, C. Diez Pardos, D. Domínguez Damiani, G. Eckerlin, D. Eckstein, T. Eichhorn, E. Eren, E. Gallo ${ }^{18}$, J. Garay Garcia, A. Geiser, J.M. Grados Luyando, A. Grohsjean, P. Gunnellini, M. Guthoff, A. Harb, J. Hauk, M. Hempel ${ }^{19}$, H. Jung, M. Kasemann, J. Keaveney, C. Kleinwort, J. Knolle, I. Korol, D. Krücker, W. Lange, A. Lelek, T. Lenz, K. Lipka, W. Lohmann ${ }^{19}$, R. Mankel, I.-A. Melzer-Pellmann, A.B. Meyer, M. Meyer, M. Missiroli, G. Mittag, J. Mnich, A. Mussgiller, D. Pitzl, A. Raspereza, M. Savitskyi, P. Saxena, R. Shevchenko, N. Stefaniuk, H. Tholen, G.P. Van Onsem, R. Walsh, Y. Wen, K. Wichmann, C. Wissing, O. Zenaiev

\section{University of Hamburg, Hamburg, Germany}

R. Aggleton, S. Bein, V. Blobel, M. Centis Vignali, T. Dreyer, E. Garutti, D. Gonzalez, J. Haller, A. Hinzmann, M. Hoffmann, A. Karavdina, G. Kasieczka, R. Klanner, R. Kogler, N. Kovalchuk, S. Kurz, D. Marconi, J. Multhaup, M. Niedziela, D. Nowatschin, T. Peiffer, A. Perieanu, A. Reimers, C. Scharf, P. Schleper, A. Schmidt, S. Schumann, J. Schwandt, J. Sonneveld, H. Stadie, G. Steinbrück, F.M. Stober, M. Stöver, D. Troendle, E. Usai, A. Vanhoefer, B. Vormwald

\section{Institut für Experimentelle Teilchenphysik, Karlsruhe, Germany}

M. Akbiyik, C. Barth, M. Baselga, S. Baur, E. Butz, R. Caspart, T. Chwalek, F. Colombo, W. De Boer, A. Dierlamm, N. Faltermann, B. Freund, R. Friese, M. Giffels, M.A. Harrendorf, F. Hartmann ${ }^{16}$, S.M. Heindl, U. Husemann, F. Kassel ${ }^{16}$, S. Kudella, H. Mildner, M.U. Mozer, Th. Müller, M. Plagge, G. Quast, K. Rabbertz, M. Schröder, I. Shvetsov, 
G. Sieber, H.J. Simonis, R. Ulrich, S. Wayand, M. Weber, T. Weiler, S. Williamson, C. Wöhrmann, R. Wolf

Institute of Nuclear and Particle Physics (INPP), NCSR Demokritos, Aghia Paraskevi, Greece

G. Anagnostou, G. Daskalakis, T. Geralis, A. Kyriakis, D. Loukas, I. Topsis-Giotis

National and Kapodistrian University of Athens, Athens, Greece

G. Karathanasis, S. Kesisoglou, A. Panagiotou, N. Saoulidou, E. Tziaferi

National Technical University of Athens, Athens, Greece

K. Kousouris, I. Papakrivopoulos

University of Ioánnina, Ioánnina, Greece

I. Evangelou, C. Foudas, P. Gianneios, P. Katsoulis, P. Kokkas, S. Mallios, N. Manthos,

I. Papadopoulos, E. Paradas, J. Strologas, F.A. Triantis, D. Tsitsonis

MTA-ELTE Lendület CMS Particle and Nuclear Physics Group, Eötvös Loránd University, Budapest, Hungary

M. Csanad, N. Filipovic, G. Pasztor, O. Surányi, G.I. Veres ${ }^{20}$

Wigner Research Centre for Physics, Budapest, Hungary

G. Bencze, C. Hajdu, D. Horvath ${ }^{21}$, Á. Hunyadi, F. Sikler, V. Veszpremi, G. Vesztergombi ${ }^{20}$, T.Á. Vámi

Institute of Nuclear Research ATOMKI, Debrecen, Hungary

N. Beni, S. Czellar, J. Karancsi ${ }^{22}$, A. Makovec, J. Molnar, Z. Szillasi

Institute of Physics, University of Debrecen, Debrecen, Hungary

M. Bartók ${ }^{20}$, P. Raics, Z.L. Trocsanyi, B. Ujvari

Indian Institute of Science (IISc), Bangalore, India

S. Choudhury, J.R. Komaragiri

National Institute of Science Education and Research, Bhubaneswar, India

S. Bahinipati ${ }^{23}$, P. Mal, K. Mandal, A. Nayak ${ }^{24}$, D.K. Sahoo ${ }^{23}$, N. Sahoo, S.K. Swain

Panjab University, Chandigarh, India

S. Bansal, S.B. Beri, V. Bhatnagar, S. Chauhan, R. Chawla, N. Dhingra, R. Gupta, A. Kaur, M. Kaur, S. Kaur, R. Kumar, P. Kumari, M. Lohan, A. Mehta, S. Sharma, J.B. Singh, G. Walia

University of Delhi, Delhi, India

Ashok Kumar, Aashaq Shah, A. Bhardwaj, B.C. Choudhary, R.B. Garg, S. Keshri, A. Kumar, S. Malhotra, M. Naimuddin, K. Ranjan, R. Sharma

Saha Institute of Nuclear Physics, HBNI, Kolkata, India

R. Bhardwaj ${ }^{25}$, R. Bhattacharya, S. Bhattacharya, U. Bhawandeep ${ }^{25}$, D. Bhowmik, S. Dey, S. Dutt ${ }^{25}$, S. Dutta, S. Ghosh, N. Majumdar, K. Mondal, S. Mukhopadhyay, S. Nandan, A. Purohit, P.K. Rout, A. Roy, S. Roy Chowdhury, S. Sarkar, M. Sharan, B. Singh, S. Thakur ${ }^{25}$ 
Indian Institute of Technology Madras, Madras, India

P.K. Behera

Bhabha Atomic Research Centre, Mumbai, India

R. Chudasama, D. Dutta, V. Jha, V. Kumar, A.K. Mohanty ${ }^{16}$, P.K. Netrakanti, L.M. Pant, P. Shukla, A. Topkar

Tata Institute of Fundamental Research-A, Mumbai, India

T. Aziz, S. Dugad, B. Mahakud, S. Mitra, G.B. Mohanty, N. Sur, B. Sutar

Tata Institute of Fundamental Research-B, Mumbai, India

S. Banerjee, S. Bhattacharya, S. Chatterjee, P. Das, M. Guchait, Sa. Jain, S. Kumar, M. Maity ${ }^{26}$, G. Majumder, K. Mazumdar, T. Sarkar ${ }^{26}$, N. Wickramage ${ }^{27}$

Indian Institute of Science Education and Research (IISER), Pune, India

S. Chauhan, S. Dube, V. Hegde, A. Kapoor, K. Kothekar, S. Pandey, A. Rane, S. Sharma

Institute for Research in Fundamental Sciences (IPM), Tehran, Iran

S. Chenarani ${ }^{28}$, E. Eskandari Tadavani, S.M. Etesami ${ }^{28}$, M. Khakzad, M. Mohammadi Najafabadi, M. Naseri, S. Paktinat Mehdiabadi ${ }^{29}$, F. Rezaei Hosseinabadi, B. Safarzadeh ${ }^{30}$, M. Zeinali

University College Dublin, Dublin, Ireland

M. Felcini, M. Grunewald

INFN Sezione di Bari ${ }^{a}$, Università di Bari ${ }^{b}$, Politecnico di Bari ${ }^{c}$, Bari, Italy M. Abbrescia ${ }^{a, b}$, C. Calabria ${ }^{a, b}$, A. Colaleo ${ }^{a}$, D. Creanza ${ }^{a, c}$, L. Cristella ${ }^{a, b}$, N. De Filippis $^{a, c}$, M. De Palma ${ }^{a, b}$, A. Di Florio ${ }^{a, b}$, F. Errico ${ }^{a, b}$, L. Fiore ${ }^{a}$, A. Gelmi ${ }^{a, b}$,

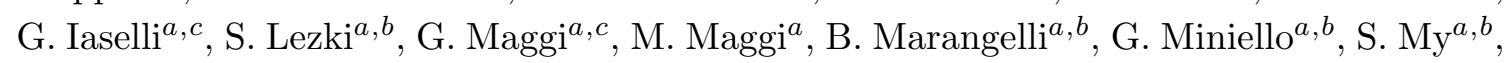
S. Nuzzo ${ }^{a, b}$, A. Pompilia ${ }^{a, b}$, G. Pugliese ${ }^{a, c}$, R. Radogna ${ }^{a}$, A. Ranieri ${ }^{a}$, G. Selvaggi ${ }^{a}, b$, A. Sharma ${ }^{a}$, L. Silvestris ${ }^{a, 16}$, R. Venditti ${ }^{a}$, P. Verwilligen ${ }^{a}$, G. Zito ${ }^{a}$

INFN Sezione di Bologna ${ }^{a}$, Università di Bologna ${ }^{b}$, Bologna, Italy

G. Abbiendi ${ }^{a}$, C. Battilana ${ }^{a, b}$, D. Bonacorsi ${ }^{a}, b$, L. Borgonovi $^{a, b}$, S. Braibant-Giacomelli ${ }^{a, b}$, R. Campanini ${ }^{a, b}$, P. Capiluppi ${ }^{a, b}$, A. Castro ${ }^{a, b}$, F.R. Cavallo ${ }^{a}$, S.S. Chhibra ${ }^{a, b}$, G. Codispoti ${ }^{a, b}$, M. Cuffiani ${ }^{a, b}$, G.M. Dallavalle ${ }^{a}$, F. Fabbri $^{a}$, A. Fanfani ${ }^{a, b}$, D. Fasanella ${ }^{a, b}$, P. Giacomelli ${ }^{a}$, C. Grandi ${ }^{a}$, L. Guiducci ${ }^{a}, b$, S. Marcellini ${ }^{a}$, G. Masetti ${ }^{a}$, A. Montanari ${ }^{a}$, F.L. Navarria ${ }^{a, b}$, F. Odorici ${ }^{a}$, A. Perrotta ${ }^{a}$, A.M. Rossi ${ }^{a, b}$, T. Rovelli ${ }^{a, b}$, G.P. Siroli ${ }^{a, b}$, N. $\operatorname{Tosi}^{a}$

INFN Sezione di Catania ${ }^{a}$, Università di Catania ${ }^{b}$, Catania, Italy

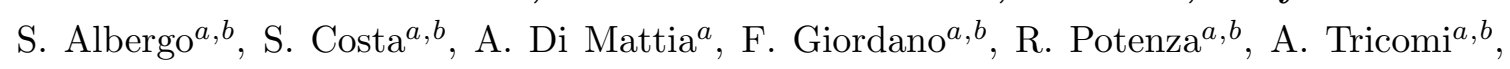
C. Tuve ${ }^{a, b}$

INFN Sezione di Firenze ${ }^{a}$, Università di Firenze ${ }^{b}$, Firenze, Italy

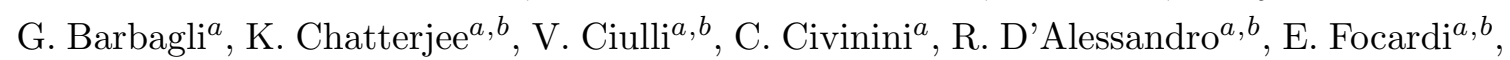
G. Latino, P. Lenzi ${ }^{a, b}$, M. Meschini ${ }^{a}$, S. Paoletti ${ }^{a}$, L. Russo $^{a}, 31$, G. Sguazzoni $^{a}$, D. Strom ${ }^{a}$, L. Viliani ${ }^{a}$ 
INFN Laboratori Nazionali di Frascati, Frascati, Italy

L. Benussi, S. Bianco, F. Fabbri, D. Piccolo, F. Primavera ${ }^{16}$

INFN Sezione di Genova ${ }^{a}$, Università di Genova ${ }^{b}$, Genova, Italy

V. Calvelli ${ }^{a}, b$, F. Ferro ${ }^{a}$, F. Ravera ${ }^{a}, b$ E. Robutti ${ }^{a}$, S. Tosi ${ }^{a, b}$

INFN Sezione di Milano-Bicocca ${ }^{a}$, Università di Milano-Bicocca ${ }^{b}$, Milano, Italy

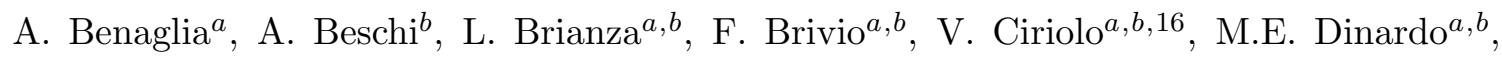
S. Fiorendi ${ }^{a, b}$, S. Gennai ${ }^{a}$, A. Ghezzi ${ }^{a, b}$, P. Govoni ${ }^{a, b}$, M. Malberti ${ }^{a, b}$, S. Malvezzi ${ }^{a}$, R.A. Manzoni ${ }^{a}, b$, D. Menasce ${ }^{a}$, L. Moroni ${ }^{a}$, M. Paganoni ${ }^{a}, b$, K. Pauwels ${ }^{a}, b$, D. Pedrini ${ }^{a}$, S. Pigazzini ${ }^{a, b, 32}$, S. Ragazzi ${ }^{a, b}$, T. Tabarelli de Fatis ${ }^{a, b}$

INFN Sezione di Napoli ${ }^{a}$, Università di Napoli 'Federico II' ${ }^{b}$, Napoli, Italy, Università della Basilicata ${ }^{c}$, Potenza, Italy, Università G. Marconi ${ }^{d}$, Roma, Italy

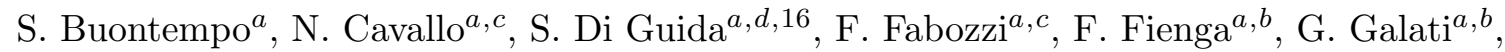
A.O.M. Iorio ${ }^{a, b}$, W.A. Khan ${ }^{a}$, L. Lista ${ }^{a}$, S. Meola ${ }^{a, d, 16}$, P. Paolucci ${ }^{a, 16}$, C. Sciacca ${ }^{a, b}$, F. Thyssen ${ }^{a}$, E. Voevodina ${ }^{a, b}$

INFN Sezione di Padova ${ }^{a}$, Università di Padova ${ }^{b}$, Padova, Italy, Università di Trento ${ }^{c}$, Trento, Italy

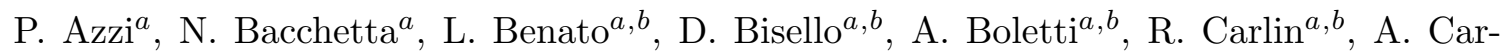
valho Antunes De Oliveira ${ }^{a, b}$, P. Checchia ${ }^{a}$, P. De Castro Manzano ${ }^{a}$, T. Dorigo ${ }^{a}$,

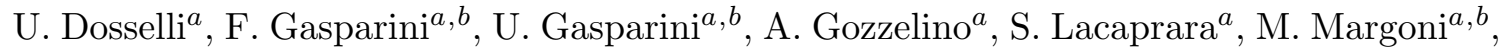
A.T. Meneguzzo ${ }^{a, b}$, N. Pozzobon ${ }^{a, b}$, P. Ronchese ${ }^{a, b}$, R. Rossin $^{a, b}$, F. Simonetto ${ }^{a, b}$, A. Tiko, E. Torassa ${ }^{a}$, M. Zanetti ${ }^{a, b}$, P. Zotto ${ }^{a, b}$, G. Zumerle ${ }^{a, b}$

INFN Sezione di Pavia ${ }^{a}$, Università di Pavia ${ }^{b}$, Pavia, Italy

A. Braghieri ${ }^{a}$, A. Magnani ${ }^{a}$, P. Montagna ${ }^{a, b}$, S.P. Ratti ${ }^{a, b}$, V. $\operatorname{Re}^{a}$, M. Ressegotti $^{a, b}$, C. Riccardi ${ }^{a, b}$, P. Salvini ${ }^{a}$, I. Vai ${ }^{a}, b$, P. Vitulo ${ }^{a, b}$

INFN Sezione di Perugia ${ }^{a}$, Università di Perugia ${ }^{b}$, Perugia, Italy

L. Alunni Solestizi ${ }^{a, b}$, M. Biasini ${ }^{a, b}$, G.M. Bilei ${ }^{a}$, C. Cecchi $^{a, b}$, D. Ciangottini ${ }^{a, b}$, L. Fanò $^{a, b}$,

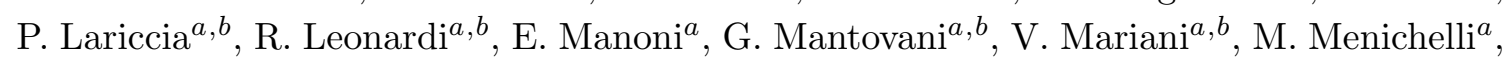
A. $\operatorname{Rossi}^{a, b}$, A. Santocchia ${ }^{a, b}$, D. Spiga ${ }^{a}$

INFN Sezione di Pisa ${ }^{a}$, Università di Pisa ${ }^{b}$, Scuola Normale Superiore di Pisa ${ }^{c}$, Pisa, Italy

K. Androsov ${ }^{a}$, P. Azzurri ${ }^{a}, 16$, G. Bagliesi ${ }^{a}$, L. Bianchini ${ }^{a}$, T. Boccali ${ }^{a}$, L. Borrello,

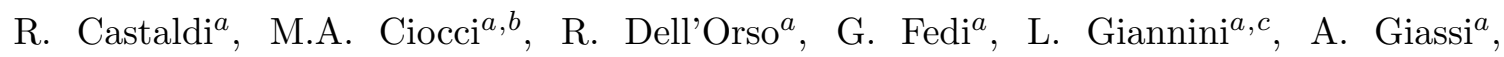
M.T. Grippo ${ }^{a, 31}$, F. Ligabue ${ }^{a, c}$, T. Lomtadze ${ }^{a}$, E. Manca ${ }^{a, c}$, G. Mandorlia ${ }^{a, c}$,

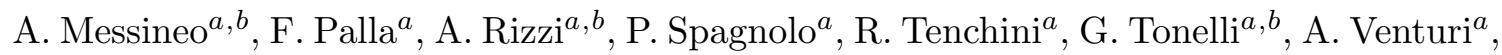
P.G. Verdini ${ }^{a}$

INFN Sezione di Roma ${ }^{a}$, Sapienza Università di Roma ${ }^{b}$, Rome, Italy

L. Barone ${ }^{a, b}$, F. Cavallari ${ }^{a}$, M. Cipriani ${ }^{a, b}$, N. Daci ${ }^{a}$, D. Del Re Re $^{a, b}$ E. Di Marco ${ }^{a, b}$, M. Diemoz ${ }^{a}$, S. Gelli ${ }^{a, b}$, E. Longo ${ }^{a, b}$, F. Margaroli ${ }^{a, b}$, B. Marzocchi ${ }^{a} b$, P. Meridiani ${ }^{a}$, 


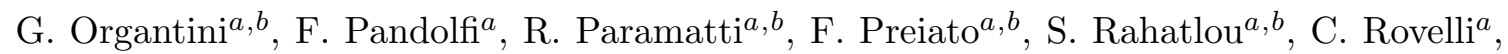
F. Santanastasio ${ }^{a, b}$

INFN Sezione di Torino ${ }^{a}$, Università di Torino ${ }^{b}$, Torino, Italy, Università del Piemonte Orientale ${ }^{c}$, Novara, Italy

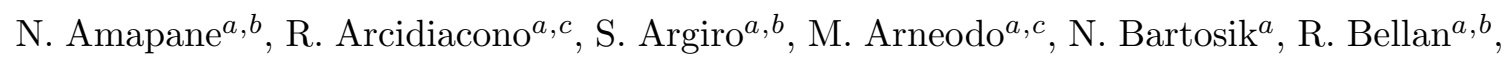

C. Biino ${ }^{a}$, N. Cartiglia ${ }^{a}$, R. Castello ${ }^{a, b}$, F. Cenna ${ }^{a, b}$, M. Costa $^{a, b}$, R. Covarelli ${ }^{a, b}$,

A. Degano ${ }^{a, b}$, N. $\operatorname{Demaria}^{a}$, B. Kiani ${ }^{a, b}$, C. Mariotti ${ }^{a}$, S. Maselli ${ }^{a}$, E. Migliore ${ }^{a, b}$,

V. Monaco ${ }^{a, b}$, E. Monteil ${ }^{a, b}$, M. Monteno ${ }^{a}$, M.M. Obertino ${ }^{a, b}$, L. Pacher $^{a, b}$, N. Pastrone ${ }^{a}$,

M. Pelliccioni ${ }^{a}$, G.L. Pinna Angioni ${ }^{a, b}$, A. Romero ${ }^{a, b}$, M. Ruspa ${ }^{a, c}$, R. Sacchi $^{a, b}$,

K. Shchelina ${ }^{a, b}$, V. Sola ${ }^{a}$, A. Solano ${ }^{a, b}$, A. Staiano ${ }^{a}$

INFN Sezione di Trieste ${ }^{a}$, Università di Trieste ${ }^{b}$, Trieste, Italy

S. Belforte ${ }^{a}$, M. Casarsa ${ }^{a}$, F. Cossutti ${ }^{a}$, G. Della Ricca ${ }^{a, b}$, A. Zanetti ${ }^{a}$

Kyungpook National University

D.H. Kim, G.N. Kim, M.S. Kim, J. Lee, S. Lee, S.W. Lee, C.S. Moon, Y.D. Oh, S. Sekmen, D.C. Son, Y.C. Yang

Chonnam National University, Institute for Universe and Elementary Particles, Kwangju, Korea

H. Kim, D.H. Moon, G. Oh

Hanyang University, Seoul, Korea

J.A. Brochero Cifuentes, J. Goh, T.J. Kim

Korea University, Seoul, Korea

S. Cho, S. Choi, Y. Go, D. Gyun, S. Ha, B. Hong, Y. Jo, Y. Kim, K. Lee, K.S. Lee, S. Lee, J. Lim, S.K. Park, Y. Roh

Seoul National University, Seoul, Korea

J. Almond, J. Kim, J.S. Kim, H. Lee, K. Lee, K. Nam, S.B. Oh, B.C. Radburn-Smith, S.h. Seo, U.K. Yang, H.D. Yoo, G.B. Yu

University of Seoul, Seoul, Korea

H. Kim, J.H. Kim, J.S.H. Lee, I.C. Park

Sungkyunkwan University, Suwon, Korea

Y. Choi, C. Hwang, J. Lee, I. Yu

Vilnius University, Vilnius, Lithuania

V. Dudenas, A. Juodagalvis, J. Vaitkus

National Centre for Particle Physics, Universiti Malaya, Kuala Lumpur, Malaysia

I. Ahmed, Z.A. Ibrahim, M.A.B. Md $\mathrm{Ali}^{33}$, F. Mohamad Idris ${ }^{34}$, W.A.T. Wan Abdullah, M.N. Yusli, Z. Zolkapli 
Centro de Investigacion y de Estudios Avanzados del IPN, Mexico City, Mexico Reyes-Almanza, R, Ramirez-Sanchez, G., Duran-Osuna, M. C., H. Castilla-Valdez, E. De La Cruz-Burelo, I. Heredia-De La Cruz ${ }^{35}$, Rabadan-Trejo, R. I., R. Lopez-Fernandez, J. Mejia Guisao, A. Sanchez-Hernandez

Universidad Iberoamericana, Mexico City, Mexico

S. Carrillo Moreno, C. Oropeza Barrera, F. Vazquez Valencia

Benemerita Universidad Autonoma de Puebla, Puebla, Mexico

J. Eysermans, I. Pedraza, H.A. Salazar Ibarguen, C. Uribe Estrada

Universidad Autónoma de San Luis Potosí, San Luis Potosí, Mexico

A. Morelos Pineda

University of Auckland, Auckland, New Zealand

D. Krofcheck

University of Canterbury, Christchurch, New Zealand

P.H. Butler

National Centre for Physics, Quaid-I-Azam University, Islamabad, Pakistan

A. Ahmad, M. Ahmad, Q. Hassan, H.R. Hoorani, A. Saddique, M.A. Shah, M. Shoaib, M. Waqas

National Centre for Nuclear Research, Swierk, Poland

H. Bialkowska, M. Bluj, B. Boimska, T. Frueboes, M. Górski, M. Kazana, K. Nawrocki, M. Szleper, P. Traczyk, P. Zalewski

Institute of Experimental Physics, Faculty of Physics, University of Warsaw, Warsaw, Poland

K. Bunkowski, A. Byszuk ${ }^{36}$, K. Doroba, A. Kalinowski, M. Konecki, J. Krolikowski, M. Misiura, M. Olszewski, A. Pyskir, M. Walczak

Laboratório de Instrumentação e Física Experimental de Partículas, Lisboa, Portugal

P. Bargassa, C. Beirão Da Cruz E Silva, A. Di Francesco, P. Faccioli, B. Galinhas, M. Gallinaro, J. Hollar, N. Leonardo, L. Lloret Iglesias, M.V. Nemallapudi, J. Seixas, G. Strong, O. Toldaiev, D. Vadruccio, J. Varela

Joint Institute for Nuclear Research, Dubna, Russia

S. Afanasiev, P. Bunin, M. Gavrilenko, I. Golutvin, I. Gorbunov, A. Kamenev, V. Karjavin, A. Lanev, A. Malakhov, V. Matveev ${ }^{37,38}$, P. Moisenz, V. Palichik, V. Perelygin, S. Shmatov, S. Shulha, N. Skatchkov, V. Smirnov, N. Voytishin, A. Zarubin

Petersburg Nuclear Physics Institute, Gatchina (St. Petersburg), Russia

Y. Ivanov, V. Kim ${ }^{39}$, E. Kuznetsova ${ }^{40}$, P. Levchenko, V. Murzin, V. Oreshkin, I. Smirnov, D. Sosnov, V. Sulimov, L. Uvarov, S. Vavilov, A. Vorobyev 
Institute for Nuclear Research, Moscow, Russia

Yu. Andreev, A. Dermenev, S. Gninenko, N. Golubev, A. Karneyeu, M. Kirsanov, N. Krasnikov, A. Pashenkov, D. Tlisov, A. Toropin

Institute for Theoretical and Experimental Physics, Moscow, Russia

V. Epshteyn, V. Gavrilov, N. Lychkovskaya, V. Popov, I. Pozdnyakov, G. Safronov, A. Spiridonov, A. Stepennov, V. Stolin, M. Toms, E. Vlasov, A. Zhokin

Moscow Institute of Physics and Technology, Moscow, Russia

T. Aushev, A. Bylinkin ${ }^{38}$

National Research Nuclear University 'Moscow Engineering Physics Institute' (MEPhI), Moscow, Russia

R. Chistov ${ }^{41}$, M. Danilov ${ }^{41}$, P. Parygin, D. Philippov, S. Polikarpov, E. Tarkovskii

P.N. Lebedev Physical Institute, Moscow, Russia

V. Andreev, M. Azarkin ${ }^{38}$, I. Dremin ${ }^{38}$, M. Kirakosyan ${ }^{38}$, S.V. Rusakov, A. Terkulov

Skobeltsyn Institute of Nuclear Physics, Lomonosov Moscow State University, Moscow, Russia

A. Baskakov, A. Belyaev, E. Boos, V. Bunichev, M. Dubinin ${ }^{42}$, L. Dudko, A. Gribushin, V. Klyukhin, O. Kodolova, I. Lokhtin, I. Miagkov, S. Obraztsov, S. Petrushanko, V. Savrin, A. Snigirev

Novosibirsk State University (NSU), Novosibirsk, Russia V. Blinov ${ }^{43}$, D. Shtol ${ }^{43}$, Y. Skovpen ${ }^{43}$

State Research Center of Russian Federation, Institute for High Energy Physics of NRC \&quot;Kurchatov Institute\&quot;, Protvino, Russia

I. Azhgirey, I. Bayshev, S. Bitioukov, D. Elumakhov, A. Godizov, V. Kachanov, A. Kalinin, D. Konstantinov, P. Mandrik, V. Petrov, R. Ryutin, A. Sobol, S. Troshin, N. Tyurin, A. Uzunian, A. Volkov

National Research Tomsk Polytechnic University, Tomsk, Russia

A. Babaev

University of Belgrade, Faculty of Physics and Vinca Institute of Nuclear Sciences, Belgrade, Serbia

P. Adzic ${ }^{44}$, P. Cirkovic, D. Devetak, M. Dordevic, J. Milosevic

Centro de Investigaciones Energéticas Medioambientales y Tecnológicas (CIEMAT), Madrid, Spain

J. Alcaraz Maestre, I. Bachiller, M. Barrio Luna, M. Cerrada, N. Colino, B. De La Cruz, A. Delgado Peris, C. Fernandez Bedoya, J.P. Fernández Ramos, J. Flix, M.C. Fouz, O. Gonzalez Lopez, S. Goy Lopez, J.M. Hernandez, M.I. Josa, D. Moran, A. Pérez-Calero Yzquierdo, J. Puerta Pelayo, I. Redondo, L. Romero, M.S. Soares, A. Triossi, A. Álvarez Fernández 
Universidad Autónoma de Madrid, Madrid, Spain

C. Albajar, J.F. de Trocóniz

\section{Universidad de Oviedo, Oviedo, Spain}

J. Cuevas, C. Erice, J. Fernandez Menendez, S. Folgueras, I. Gonzalez Caballero, J.R. González Fernández, E. Palencia Cortezon, S. Sanchez Cruz, P. Vischia, J.M. Vizan Garcia

Instituto de Física de Cantabria (IFCA), CSIC-Universidad de Cantabria, Santander, Spain

I.J. Cabrillo, A. Calderon, B. Chazin Quero, J. Duarte Campderros, M. Fernandez, P.J. Fernández Manteca, J. Garcia-Ferrero, A. García Alonso, G. Gomez, A. Lopez Virto, J. Marco, C. Martinez Rivero, P. Martinez Ruiz del Arbol, F. Matorras, J. Piedra Gomez, C. Prieels, T. Rodrigo, A. Ruiz-Jimeno, L. Scodellaro, N. Trevisani, I. Vila, R. Vilar Cortabitarte

\section{CERN, European Organization for Nuclear Research, Geneva, Switzerland}

D. Abbaneo, B. Akgun, E. Auffray, P. Baillon, A.H. Ball, D. Barney, J. Bendavid, M. Bianco, A. Bocci, C. Botta, T. Camporesi, M. Cepeda, G. Cerminara, E. Chapon, Y. Chen, D. d'Enterria, A. Dabrowski, V. Daponte, A. David, M. De Gruttola, A. De Roeck, N. Deelen, M. Dobson, T. du Pree, M. Dünser, N. Dupont, A. Elliott-Peisert, P. Everaerts, F. Fallavollita ${ }^{45}$, G. Franzoni, J. Fulcher, W. Funk, D. Gigi, A. Gilbert, K. Gill, F. Glege, D. Gulhan, J. Hegeman, V. Innocente, A. Jafari, P. Janot, O. Karacheban ${ }^{19}$, J. Kieseler, V. Knünz, A. Kornmayer, M. Krammer ${ }^{1}$, C. Lange, P. Lecoq, C. Lourenço, M.T. Lucchini, L. Malgeri, M. Mannelli, A. Martelli, F. Meijers, J.A. Merlin, S. Mersi, E. Meschi, P. Milenovic ${ }^{46}$, F. Moortgat, M. Mulders, H. Neugebauer, J. Ngadiuba, S. Orfanelli, L. Orsini, F. Pantaleo ${ }^{16}$, L. Pape, E. Perez, M. Peruzzi, A. Petrilli, G. Petrucciani, A. Pfeiffer, M. Pierini, F.M. Pitters, D. Rabady, A. Racz, T. Reis, G. Rolandi ${ }^{47}$, M. Rovere, H. Sakulin, C. Schäfer, C. Schwick, M. Seidel, M. Selvaggi, A. Sharma, P. Silva, P. Sphicas ${ }^{48}$, A. Stakia, J. Steggemann, M. Stoye, M. Tosi, D. Treille, A. Tsirou, V. Veckalns ${ }^{49}$, M. Verweij, W.D. Zeuner

\section{Paul Scherrer Institut, Villigen, Switzerland}

W. Bertl ${ }^{\dagger}$, L. Caminada ${ }^{50}$, K. Deiters, W. Erdmann, R. Horisberger, Q. Ingram, H.C. Kaestli, D. Kotlinski, U. Langenegger, T. Rohe, S.A. Wiederkehr

ETH Zurich - Institute for Particle Physics and Astrophysics (IPA), Zurich, Switzerland

M. Backhaus, L. Bäni, P. Berger, B. Casal, N. Chernyavskaya, G. Dissertori, M. Dittmar, M. Donegà, C. Dorfer, C. Grab, C. Heidegger, D. Hits, J. Hoss, T. Klijnsma, W. Lustermann, M. Marionneau, M.T. Meinhard, D. Meister, F. Micheli, P. Musella, F. NessiTedaldi, J. Pata, F. Pauss, G. Perrin, L. Perrozzi, M. Quittnat, M. Reichmann, D. Ruini, D.A. Sanz Becerra, M. Schönenberger, L. Shchutska, V.R. Tavolaro, K. Theofilatos, M.L. Vesterbacka Olsson, R. Wallny, D.H. Zhu 
Universität Zürich, Zurich, Switzerland

T.K. Aarrestad, C. Amsler ${ }^{51}$, D. Brzhechko, M.F. Canelli, A. De Cosa, R. Del Burgo, S. Donato, C. Galloni, T. Hreus, B. Kilminster, I. Neutelings, D. Pinna, G. Rauco, P. Robmann, D. Salerno, K. Schweiger, C. Seitz, Y. Takahashi, A. Zucchetta

National Central University, Chung-Li, Taiwan

V. Candelise, Y.H. Chang, K.y. Cheng, T.H. Doan, Sh. Jain, R. Khurana, C.M. Kuo, W. Lin, A. Pozdnyakov, S.S. Yu

National Taiwan University (NTU), Taipei, Taiwan

Arun Kumar, P. Chang, Y. Chao, K.F. Chen, P.H. Chen, F. Fiori, W.-S. Hou, Y. Hsiung, Y.F. Liu, R.-S. Lu, E. Paganis, A. Psallidas, A. Steen, J.f. Tsai

Chulalongkorn University, Faculty of Science, Department of Physics, Bangkok, Thailand

B. Asavapibhop, K. Kovitanggoon, G. Singh, N. Srimanobhas

Çukurova University, Physics Department, Science and Art Faculty, Adana, Turkey

A. Bat, F. Boran, S. Cerci ${ }^{52}$, S. Damarseckin, Z.S. Demiroglu, C. Dozen, I. Dumanoglu,

S. Girgis, G. Gokbulut, Y. Guler, I. $\operatorname{Hos}^{53}$, E.E. Kangal ${ }^{54}$, O. Kara, A. Kayis Topaksu,

U. Kiminsu, M. Oglakci, G. Onengut, K. Ozdemir ${ }^{55}$, D. Sunar Cerci ${ }^{52}$, U.G. Tok,

H. Topakli ${ }^{56}$, S. Turkcapar, I.S. Zorbakir, C. Zorbilmez

Middle East Technical University, Physics Department, Ankara, Turkey

G. Karapinar ${ }^{57}$, K. Ocalan ${ }^{58}$, M. Yalvac, M. Zeyrek

Bogazici University, Istanbul, Turkey

E. Gülmez, M. Kaya ${ }^{59}$, O. Kaya ${ }^{60}$, S. Tekten, E.A. Yetkin ${ }^{61}$

Istanbul Technical University, Istanbul, Turkey

M.N. Agaras, S. Atay, A. Cakir, K. Cankocak, Y. Komurcu

Institute for Scintillation Materials of National Academy of Science of Ukraine, Kharkov, Ukraine

B. Grynyov

National Scientific Center, Kharkov Institute of Physics and Technology, Kharkov, Ukraine

L. Levchuk

University of Bristol, Bristol, United Kingdom

F. Ball, L. Beck, J.J. Brooke, D. Burns, E. Clement, D. Cussans, O. Davignon, H. Flacher,

J. Goldstein, G.P. Heath, H.F. Heath, L. Kreczko, D.M. Newbold ${ }^{62}$, S. Paramesvaran,

T. Sakuma, S. Seif El Nasr-storey, D. Smith, V.J. Smith 


\section{Rutherford Appleton Laboratory, Didcot, United Kingdom}

K.W. Bell, A. Belyaev ${ }^{63}$, C. Brew, R.M. Brown, D. Cieri, D.J.A. Cockerill, J.A. Coughlan, K. Harder, S. Harper, J. Linacre, E. Olaiya, D. Petyt, C.H. Shepherd-Themistocleous, A. Thea, I.R. Tomalin, T. Williams, W.J. Womersley

\section{Imperial College, London, United Kingdom}

G. Auzinger, R. Bainbridge, P. Bloch, J. Borg, S. Breeze, O. Buchmuller, A. Bundock, S. Casasso, D. Colling, L. Corpe, P. Dauncey, G. Davies, M. Della Negra, R. Di Maria, A. Elwood, Y. Haddad, G. Hall, G. Iles, T. James, M. Komm, R. Lane, C. Laner, L. Lyons, A.-M. Magnan, S. Malik, L. Mastrolorenzo, T. Matsushita, J. $\mathrm{Nash}^{64}$, A. Nikitenko ${ }^{7}$, V. Palladino, M. Pesaresi, A. Richards, A. Rose, E. Scott, C. Seez, A. Shtipliyski, T. Strebler, S. Summers, A. Tapper, K. Uchida, M. Vazquez Acosta ${ }^{65}$, T. Virdee ${ }^{16}$, N. Wardle, D. Winterbottom, J. Wright, S.C. Zenz

Brunel University, Uxbridge, United Kingdom

J.E. Cole, P.R. Hobson, A. Khan, P. Kyberd, A. Morton, I.D. Reid, L. Teodorescu, S. Zahid Baylor University, Waco, U.S.A.

A. Borzou, K. Call, J. Dittmann, K. Hatakeyama, H. Liu, N. Pastika, C. Smith

Catholic University of America, Washington DC, U.S.A.

R. Bartek, A. Dominguez

The University of Alabama, Tuscaloosa, U.S.A.

A. Buccilli, S.I. Cooper, C. Henderson, P. Rumerio, C. West

Boston University, Boston, U.S.A.

D. Arcaro, A. Avetisyan, T. Bose, D. Gastler, D. Rankin, C. Richardson, J. Rohlf, L. Sulak, D. Zou

\section{Brown University, Providence, U.S.A.}

G. Benelli, D. Cutts, M. Hadley, J. Hakala, U. Heintz, J.M. Hogan ${ }^{66}$, K.H.M. Kwok, E. Laird, G. Landsberg, J. Lee, Z. Mao, M. Narain, J. Pazzini, S. Piperov, S. Sagir, R. Syarif, D. Yu

University of California, Davis, Davis, U.S.A.

R. Band, C. Brainerd, R. Breedon, D. Burns, M. Calderon De La Barca Sanchez, M. Chertok, J. Conway, R. Conway, P.T. Cox, R. Erbacher, C. Flores, G. Funk, W. Ko, R. Lander, C. Mclean, M. Mulhearn, D. Pellett, J. Pilot, S. Shalhout, M. Shi, J. Smith, D. Stolp, D. Taylor, K. Tos, M. Tripathi, Z. Wang, F. Zhang

University of California, Los Angeles, U.S.A.

M. Bachtis, C. Bravo, R. Cousins, A. Dasgupta, A. Florent, J. Hauser, M. Ignatenko, N. Mccoll, S. Regnard, D. Saltzberg, C. Schnaible, V. Valuev

University of California, Riverside, Riverside, U.S.A.

E. Bouvier, K. Burt, R. Clare, J. Ellison, J.W. Gary, S.M.A. Ghiasi Shirazi, G. Hanson, G. Karapostoli, E. Kennedy, F. Lacroix, O.R. Long, M. Olmedo Negrete, M.I. Paneva, W. Si, L. Wang, H. Wei, S. Wimpenny, B. R. Yates 
University of California, San Diego, La Jolla, U.S.A.

J.G. Branson, S. Cittolin, M. Derdzinski, R. Gerosa, D. Gilbert, B. Hashemi, A. Holzner, D. Klein, G. Kole, V. Krutelyov, J. Letts, M. Masciovecchio, D. Olivito, S. Padhi, M. Pieri, M. Sani, V. Sharma, S. Simon, M. Tadel, A. Vartak, S. Wasserbaech ${ }^{67}$, J. Wood, F. Würthwein, A. Yagil, G. Zevi Della Porta

University of California, Santa Barbara - Department of Physics, Santa Barbara, U.S.A.

N. Amin, R. Bhandari, J. Bradmiller-Feld, C. Campagnari, M. Citron, A. Dishaw, V. Dutta, M. Franco Sevilla, L. Gouskos, R. Heller, J. Incandela, A. Ovcharova, H. Qu, J. Richman, D. Stuart, I. Suarez, J. Yoo

California Institute of Technology, Pasadena, U.S.A.

D. Anderson, A. Bornheim, J. Bunn, J.M. Lawhorn, H.B. Newman, T. Q. Nguyen, C. Pena, M. Spiropulu, J.R. Vlimant, R. Wilkinson, S. Xie, Z. Zhang, R.Y. Zhu

Carnegie Mellon University, Pittsburgh, U.S.A.

M.B. Andrews, T. Ferguson, T. Mudholkar, M. Paulini, J. Russ, M. Sun, H. Vogel, I. Vorobiev, M. Weinberg

University of Colorado Boulder, Boulder, U.S.A.

J.P. Cumalat, W.T. Ford, F. Jensen, A. Johnson, M. Krohn, S. Leontsinis, E. MacDonald, T. Mulholland, K. Stenson, K.A. Ulmer, S.R. Wagner

Cornell University, Ithaca, U.S.A.

J. Alexander, J. Chaves, Y. Cheng, J. Chu, A. Datta, K. Mcdermott, N. Mirman, J.R. Patterson, D. Quach, A. Rinkevicius, A. Ryd, L. Skinnari, L. Soffi, S.M. Tan, Z. Tao, J. Thom, J. Tucker, P. Wittich, M. Zientek

Fermi National Accelerator Laboratory, Batavia, U.S.A.

S. Abdullin, M. Albrow, M. Alyari, G. Apollinari, A. Apresyan, A. Apyan, S. Banerjee, L.A.T. Bauerdick, A. Beretvas, J. Berryhill, P.C. Bhat, G. Bolla ${ }^{\dagger}$, K. Burkett, J.N. Butler, A. Canepa, G.B. Cerati, H.W.K. Cheung, F. Chlebana, M. Cremonesi, J. Duarte, V.D. Elvira, J. Freeman, Z. Gecse, E. Gottschalk, L. Gray, D. Green, S. Grünendahl, O. Gutsche, J. Hanlon, R.M. Harris, S. Hasegawa, J. Hirschauer, Z. Hu, B. Jayatilaka, S. Jindariani, M. Johnson, U. Joshi, B. Klima, M.J. Kortelainen, B. Kreis, S. Lammel, D. Lincoln, R. Lipton, M. Liu, T. Liu, R. Lopes De Sá, J. Lykken, K. Maeshima, N. Magini, J.M. Marraffino, D. Mason, P. McBride, P. Merkel, S. Mrenna, S. Nahn, V. O'Dell, K. Pedro, O. Prokofyev, G. Rakness, L. Ristori, A. Savoy-Navarro ${ }^{68}$, B. Schneider, E. Sexton-Kennedy, A. Soha, W.J. Spalding, L. Spiegel, S. Stoynev, J. Strait, N. Strobbe, L. Taylor, S. Tkaczyk, N.V. Tran, L. Uplegger, E.W. Vaandering, C. Vernieri, M. Verzocchi, R. Vidal, M. Wang, H.A. Weber, A. Whitbeck, W. Wu

University of Florida, Gainesville, U.S.A.

D. Acosta, P. Avery, P. Bortignon, D. Bourilkov, A. Brinkerhoff, A. Carnes, M. Carver, D. Curry, R.D. Field, I.K. Furic, S.V. Gleyzer, B.M. Joshi, J. Konigsberg, A. Korytov, 
K. Kotov, P. Ma, K. Matchev, H. Mei, G. Mitselmakher, K. Shi, D. Sperka, N. Terentyev, L. Thomas, J. Wang, S. Wang, J. Yelton

Florida International University, Miami, U.S.A.

Y.R. Joshi, S. Linn, P. Markowitz, J.L. Rodriguez

Florida State University, Tallahassee, U.S.A.

A. Ackert, T. Adams, A. Askew, S. Hagopian, V. Hagopian, K.F. Johnson, T. Kolberg, G. Martinez, T. Perry, H. Prosper, A. Saha, A. Santra, V. Sharma, R. Yohay

Florida Institute of Technology, Melbourne, U.S.A.

M.M. Baarmand, V. Bhopatkar, S. Colafranceschi, M. Hohlmann, D. Noonan, T. Roy, F. Yumiceva

University of Illinois at Chicago (UIC), Chicago, U.S.A.

M.R. Adams, L. Apanasevich, D. Berry, R.R. Betts, R. Cavanaugh, X. Chen, S. Dittmer, O. Evdokimov, C.E. Gerber, D.A. Hangal, D.J. Hofman, K. Jung, J. Kamin, I.D. Sandoval Gonzalez, M.B. Tonjes, N. Varelas, H. Wang, Z. Wu, J. Zhang

The University of Iowa, Iowa City, U.S.A.

B. Bilki ${ }^{69}$, W. Clarida, K. Dilsiz ${ }^{70}$, S. Durgut, R.P. Gandrajula, M. Haytmyradov, V. Khristenko, J.-P. Merlo, H. Mermerkaya ${ }^{71}$, A. Mestvirishvili, A. Moeller, J. Nachtman, H. Ogul ${ }^{72}$, Y. Onel, F. Ozok ${ }^{73}$, A. Penzo, C. Snyder, E. Tiras, J. Wetzel, K. Yi

Johns Hopkins University, Baltimore, U.S.A.

B. Blumenfeld, A. Cocoros, N. Eminizer, D. Fehling, L. Feng, A.V. Gritsan, P. Maksimovic, J. Roskes, U. Sarica, M. Swartz, M. Xiao, C. You

The University of Kansas, Lawrence, U.S.A.

A. Al-bataineh, P. Baringer, A. Bean, S. Boren, J. Bowen, J. Castle, S. Khalil, A. Kropivnitskaya, D. Majumder, W. Mcbrayer, M. Murray, C. Rogan, C. Royon, S. Sanders, E. Schmitz, J.D. Tapia Takaki, Q. Wang

Kansas State University, Manhattan, U.S.A.

A. Ivanov, K. Kaadze, Y. Maravin, A. Modak, A. Mohammadi, L.K. Saini, N. Skhirtladze

Lawrence Livermore National Laboratory, Livermore, U.S.A.

F. Rebassoo, D. Wright

University of Maryland, College Park, U.S.A.

A. Baden, O. Baron, A. Belloni, S.C. Eno, Y. Feng, C. Ferraioli, N.J. Hadley, S. Jabeen, G.Y. Jeng, R.G. Kellogg, J. Kunkle, A.C. Mignerey, F. Ricci-Tam, Y.H. Shin, A. Skuja, S.C. Tonwar

\section{Massachusetts Institute of Technology, Cambridge, U.S.A.}

D. Abercrombie, B. Allen, V. Azzolini, R. Barbieri, A. Baty, G. Bauer, R. Bi, S. Brandt, W. Busza, I.A. Cali, M. D’Alfonso, Z. Demiragli, G. Gomez Ceballos, M. Goncharov, P. Harris, D. Hsu, M. Hu, Y. Iiyama, G.M. Innocenti, M. Klute, D. Kovalskyi, Y.-J. Lee, A. Levin, P.D. Luckey, B. Maier, A.C. Marini, C. Mcginn, C. Mironov, S. Narayanan, 
X. Niu, C. Paus, C. Roland, G. Roland, G.S.F. Stephans, K. Sumorok, K. Tatar,

D. Velicanu, J. Wang, T.W. Wang, B. Wyslouch, S. Zhaozhong

University of Minnesota, Minneapolis, U.S.A.

A.C. Benvenuti, R.M. Chatterjee, A. Evans, P. Hansen, S. Kalafut, Y. Kubota, Z. Lesko, J. Mans, S. Nourbakhsh, N. Ruckstuhl, R. Rusack, J. Turkewitz, M.A. Wadud

University of Mississippi, Oxford, U.S.A.

J.G. Acosta, S. Oliveros

University of Nebraska-Lincoln, Lincoln, U.S.A.

E. Avdeeva, K. Bloom, D.R. Claes, C. Fangmeier, F. Golf, R. Gonzalez Suarez, R. Kamalieddin, I. Kravchenko, J. Monroy, J.E. Siado, G.R. Snow, B. Stieger

State University of New York at Buffalo, Buffalo, U.S.A.

J. Dolen, A. Godshalk, C. Harrington, I. Iashvili, D. Nguyen, A. Parker, S. Rappoccio, B. Roozbahani

Northeastern University, Boston, U.S.A.

G. Alverson, E. Barberis, C. Freer, A. Hortiangtham, A. Massironi, D.M. Morse, T. Orimoto, R. Teixeira De Lima, T. Wamorkar, B. Wang, A. Wisecarver, D. Wood

Northwestern University, Evanston, U.S.A.

S. Bhattacharya, O. Charaf, K.A. Hahn, N. Mucia, N. Odell, M.H. Schmitt, K. Sung, M. Trovato, M. Velasco

University of Notre Dame, Notre Dame, U.S.A.

R. Bucci, N. Dev, M. Hildreth, K. Hurtado Anampa, C. Jessop, D.J. Karmgard, N. Kellams, K. Lannon, W. Li, N. Loukas, N. Marinelli, F. Meng, C. Mueller, Y. Musienko ${ }^{37}$, M. Planer, A. Reinsvold, R. Ruchti, P. Siddireddy, G. Smith, S. Taroni, M. Wayne, A. Wightman, M. Wolf, A. Woodard

The Ohio State University, Columbus, U.S.A.

J. Alimena, L. Antonelli, B. Bylsma, L.S. Durkin, S. Flowers, B. Francis, A. Hart, C. Hill, W. Ji, T.Y. Ling, W. Luo, B.L. Winer, H.W. Wulsin

Princeton University, Princeton, U.S.A.

S. Cooperstein, O. Driga, P. Elmer, J. Hardenbrook, P. Hebda, S. Higginbotham, A. Kalogeropoulos, D. Lange, J. Luo, D. Marlow, K. Mei, I. Ojalvo, J. Olsen, C. Palmer, P. Piroué, J. Salfeld-Nebgen, D. Stickland, C. Tully

University of Puerto Rico, Mayaguez, U.S.A.

S. Malik, S. Norberg

Purdue University, West Lafayette, U.S.A.

A. Barker, V.E. Barnes, S. Das, L. Gutay, M. Jones, A.W. Jung, A. Khatiwada, D.H. Miller, N. Neumeister, C.C. Peng, H. Qiu, J.F. Schulte, J. Sun, F. Wang, R. Xiao, W. Xie

Purdue University Northwest, Hammond, U.S.A.

T. Cheng, N. Parashar 
Rice University, Houston, U.S.A.

Z. Chen, K.M. Ecklund, S. Freed, F.J.M. Geurts, M. Guilbaud, M. Kilpatrick, W. Li, B. Michlin, B.P. Padley, J. Roberts, J. Rorie, W. Shi, Z. Tu, J. Zabel, A. Zhang

University of Rochester, Rochester, U.S.A.

A. Bodek, P. de Barbaro, R. Demina, Y.t. Duh, T. Ferbel, M. Galanti, A. Garcia-Bellido, J. Han, O. Hindrichs, A. Khukhunaishvili, K.H. Lo, P. Tan, M. Verzetti

The Rockefeller University, New York, U.S.A.

R. Ciesielski, K. Goulianos, C. Mesropian

Rutgers, The State University of New Jersey, Piscataway, U.S.A.

A. Agapitos, J.P. Chou, Y. Gershtein, T.A. Gómez Espinosa, E. Halkiadakis, M. Heindl, E. Hughes, S. Kaplan, R. Kunnawalkam Elayavalli, S. Kyriacou, A. Lath, R. Montalvo, K. Nash, M. Osherson, H. Saka, S. Salur, S. Schnetzer, D. Sheffield, S. Somalwar, R. Stone, S. Thomas, P. Thomassen, M. Walker

University of Tennessee, Knoxville, U.S.A.

A.G. Delannoy, J. Heideman, G. Riley, K. Rose, S. Spanier, K. Thapa

Texas A\&M University, College Station, U.S.A.

O. Bouhali ${ }^{74}$, A. Castaneda Hernandez ${ }^{74}$, A. Celik, M. Dalchenko, M. De Mattia, A. Delgado, S. Dildick, R. Eusebi, J. Gilmore, T. Huang, T. Kamon ${ }^{75}$, R. Mueller, Y. Pakhotin, R. Patel, A. Perloff, L. Perniè, D. Rathjens, A. Safonov, A. Tatarinov

Texas Tech University, Lubbock, U.S.A.

N. Akchurin, J. Damgov, F. De Guio, P.R. Dudero, J. Faulkner, E. Gurpinar, S. Kunori, K. Lamichhane, S.W. Lee, T. Mengke, S. Muthumuni, T. Peltola, S. Undleeb, I. Volobouev, Z. Wang

Vanderbilt University, Nashville, U.S.A.

S. Greene, A. Gurrola, R. Janjam, W. Johns, C. Maguire, A. Melo, H. Ni, K. Padeken, J.D. Ruiz Alvarez, P. Sheldon, S. Tuo, J. Velkovska, Q. Xu

University of Virginia, Charlottesville, U.S.A.

M.W. Arenton, P. Barria, B. Cox, R. Hirosky, M. Joyce, A. Ledovskoy, H. Li, C. Neu, T. Sinthuprasith, Y. Wang, E. Wolfe, F. Xia

Wayne State University, Detroit, U.S.A.

R. Harr, P.E. Karchin, N. Poudyal, J. Sturdy, P. Thapa, S. Zaleski

University of Wisconsin - Madison, Madison, WI, U.S.A.

M. Brodski, J. Buchanan, C. Caillol, D. Carlsmith, S. Dasu, L. Dodd, S. Duric, B. Gomber, M. Grothe, M. Herndon, A. Hervé, U. Hussain, P. Klabbers, A. Lanaro, A. Levine, K. Long, R. Loveless, V. Rekovic, T. Ruggles, A. Savin, N. Smith, W.H. Smith, N. Woods

$\dagger$ : Deceased

1: Also at Vienna University of Technology, Vienna, Austria 
2: Also at IRFU, CEA, Université Paris-Saclay, Gif-sur-Yvette, France

3: Also at Universidade Estadual de Campinas, Campinas, Brazil

4: Also at Federal University of Rio Grande do Sul, Porto Alegre, Brazil

5: Also at Universidade Federal de Pelotas, Pelotas, Brazil

6: Also at Université Libre de Bruxelles, Bruxelles, Belgium

7: Also at Institute for Theoretical and Experimental Physics, Moscow, Russia

8: Also at Joint Institute for Nuclear Research, Dubna, Russia

9: Also at Fayoum University, El-Fayoum, Egypt

10: Now at British University in Egypt, Cairo, Egypt

11: Now at Ain Shams University, Cairo, Egypt

12: Also at Department of Physics, King Abdulaziz University, Jeddah, Saudi Arabia

13: Also at Université de Haute Alsace, Mulhouse, France

14: Also at Skobeltsyn Institute of Nuclear Physics, Lomonosov Moscow State University, Moscow, Russia

15: Also at Tbilisi State University, Tbilisi, Georgia

16: Also at CERN, European Organization for Nuclear Research, Geneva, Switzerland

17: Also at RWTH Aachen University, III. Physikalisches Institut A, Aachen, Germany

18: Also at University of Hamburg, Hamburg, Germany

19: Also at Brandenburg University of Technology, Cottbus, Germany

20: Also at MTA-ELTE Lendület CMS Particle and Nuclear Physics Group, Eötvös Loránd University, Budapest, Hungary

21: Also at Institute of Nuclear Research ATOMKI, Debrecen, Hungary

22: Also at Institute of Physics, University of Debrecen, Debrecen, Hungary

23: Also at Indian Institute of Technology Bhubaneswar, Bhubaneswar, India

24: Also at Institute of Physics, Bhubaneswar, India

25: Also at Shoolini University, Solan, India

26: Also at University of Visva-Bharati, Santiniketan, India

27: Also at University of Ruhuna, Matara, Sri Lanka

28: Also at Isfahan University of Technology, Isfahan, Iran

29: Also at Yazd University, Yazd, Iran

30: Also at Plasma Physics Research Center, Science and Research Branch, Islamic Azad University, Tehran, Iran

31: Also at Università degli Studi di Siena, Siena, Italy

32: Also at INFN Sezione di Milano-Bicocca; Università di Milano-Bicocca, Milano, Italy

33: Also at International Islamic University of Malaysia, Kuala Lumpur, Malaysia

34: Also at Malaysian Nuclear Agency, MOSTI, Kajang, Malaysia

35: Also at Consejo Nacional de Ciencia y Tecnología, Mexico city, Mexico

36: Also at Warsaw University of Technology, Institute of Electronic Systems, Warsaw, Poland

37: Also at Institute for Nuclear Research, Moscow, Russia

38: Now at National Research Nuclear University 'Moscow Engineering Physics Institute' (MEPhI), Moscow, Russia

39: Also at St. Petersburg State Polytechnical University, St. Petersburg, Russia

40: Also at University of Florida, Gainesville, U.S.A.

41: Also at P.N. Lebedev Physical Institute, Moscow, Russia

42: Also at California Institute of Technology, Pasadena, U.S.A.

43: Also at Budker Institute of Nuclear Physics, Novosibirsk, Russia

44: Also at Faculty of Physics, University of Belgrade, Belgrade, Serbia

45: Also at INFN Sezione di Pavia; Università di Pavia, Pavia, Italy 
46: Also at University of Belgrade, Faculty of Physics and Vinca Institute of Nuclear Sciences, Belgrade, Serbia

47: Also at Scuola Normale e Sezione dell'INFN, Pisa, Italy

48: Also at National and Kapodistrian University of Athens, Athens, Greece

49: Also at Riga Technical University, Riga, Latvia

50: Also at Universität Zürich, Zurich, Switzerland

51: Also at Stefan Meyer Institute for Subatomic Physics (SMI), Vienna, Austria

52: Also at Adiyaman University, Adiyaman, Turkey

53: Also at Istanbul Aydin University, Istanbul, Turkey

54: Also at Mersin University, Mersin, Turkey

55: Also at Piri Reis University, Istanbul, Turkey

56: Also at Gaziosmanpasa University, Tokat, Turkey

57: Also at Izmir Institute of Technology, Izmir, Turkey

58: Also at Necmettin Erbakan University, Konya, Turkey

59: Also at Marmara University, Istanbul, Turkey

60: Also at Kafkas University, Kars, Turkey

61: Also at Istanbul Bilgi University, Istanbul, Turkey

62: Also at Rutherford Appleton Laboratory, Didcot, United Kingdom

63: Also at School of Physics and Astronomy, University of Southampton, Southampton, United Kingdom

64: Also at Monash University, Faculty of Science, Clayton, Australia

65: Also at Instituto de Astrofísica de Canarias, La Laguna, Spain

66: Also at Bethel University, ST. PAUL, U.S.A.

67: Also at Utah Valley University, Orem, U.S.A.

68: Also at Purdue University, West Lafayette, U.S.A.

69: Also at Beykent University, Istanbul, Turkey

70: Also at Bingol University, Bingol, Turkey

71: Also at Erzincan University, Erzincan, Turkey

72: Also at Sinop University, Sinop, Turkey

73: Also at Mimar Sinan University, Istanbul, Istanbul, Turkey

74: Also at Texas A\&M University at Qatar, Doha, Qatar

75: Also at Kyungpook National University, Daegu, Korea 